\title{
INTERPOSITION AND LATTICE CONES OF FUNCTIONS
}

\author{
BY
}

\author{
JÖRG BLATTER AND G. L. SEEVER( $\left.{ }^{1}\right)$
}

\begin{abstract}
A lattice cone of functions on a set $X$ is a convex cone of bounded real-valued functions on $X$ which contains the constants and which is closed under the lattice operations. Our principal results concern the relation between closed lattice cones on a set $X$ and certain binary relations, called inclusions, on the power set of $X$. These results are applied to interposition problems, Császár compactifications of quasi-proximity spaces, the compactification of Nachbin's completely regular ordered topological spaces, and a problem in best approximation.
\end{abstract}

1. Introduction and basic definitions. Let $X$ be a set, and let $K, L$ and $M$ be subsets of $B(X)$, the space of all bounded real-valued functions on $X$. We say that $K$ interposes the pair $(L, M)$ if whenever $f \in L, g \in M$ and $f \leqslant g$ (i.e. $f(x)$ $\leqslant g(x)$ for all $x \in X)$ there is an $h \in K$ such that $f \leqslant h \leqslant g$. In the case that $K$, $L$ and $M$ are closed lattice cones (of functions) on $X$, that is, closed( $\left.{ }^{2}\right)$ convex cones in $B(X)$ which contain the constant functions and are closed under the lattice operations, we shall obtain a condition necessary and sufficient for $K$ to interpose $(L, M)$. This condition is stated in terms of certain binary relations, called inclusions, on $2^{X}$ ( $=$ set of all subsets of $X$ ) which are associated with $K$, $L$ and $M$. An inclusion $\left({ }^{3}\right)$ on $X$ is a binary relation $<$ on $2^{X}$ which satisfies:

(I $)$ For all $A \subset X, \varnothing \prec A$ and $A \prec X$,

(I) $A \prec B \Rightarrow A \subset B$,

Received by the editors June $11,1974$.

AMS (MOS) subject classifications (1970). Primary 46E05; Secondary 54E05, 54F05, 41 A50.

Key words and phrases. Lattice cone, inclusion, interposition, quasi-proximity, ordered topological space, quasi-proximity compactification, ordered topological space compactification, best approximation.

(1) During the preparation of portions of this paper, the first-named author was supported by the Conselho Nacional de Pesquisas, Rio de Janeiro and the Gesellschaft für Mathematik und Datenverarbeitung, Bonn; and the second-named author by the Sonderforschungsbereich $\mathbf{7 2}$ at the Institut für Angewandte Mathematik der Universităt Bonn and by National Science Foundation Grant GP-38539.

(2) $B(X)$ is always taken to be equipped with the topology of uniform convergence.

$\left({ }^{3}\right)$ This term is of our own coinage. Inclusions, under the name topogenous orders, and idempotent inclusions, under the name topogenous structures, were introduced by Császár who has investigated them in great detail $[6]$, although from a different point of view than ours. In particular, portions of the Characterization Theorem (below) are implicit in Chapter 12 of $[6]$. 
( $\left.\mathrm{I}_{3}\right) A \cup B \prec C \Leftrightarrow A \prec C$ and $B \prec C$,

$\left(\mathrm{I}_{4}\right) C \prec A \cap B \Leftrightarrow C \prec A$ and $C \prec B$, and $\prec$ is called an idempotent inclusion if it also satisfies

$\left(\mathrm{I}_{5}\right) A \prec B \Rightarrow \exists C \subset X \ni A \prec C$ and $C \prec B$.

An inclusion $\prec$ on $X$ induces on $B(X)$ a binary relation, also denoted by $\prec$, as follows.

$f<g \Leftrightarrow\{x: f(x) \geqslant s\}<\{x: g(x) \geqslant r\}$ whenever $r, s \in \mathbf{R}, r<s$. Note that for $A, B \subset X, A \prec B$ iff $1_{A} \prec 1_{B}$ (= characteristic function of $B$ ). We denote by $C(X, \prec)$ the set of all $f \in B(X)$ such that $f<f$. Our basic result concerning interposition is the

INTERPOSITION THEOREM. Let $X$ be a set and let $\prec$ be an inclusion on $X$. Then the following conditions are equivalent.

(a) $\prec$ is idempotent.

(b) For $f, g \in B(X)$, whenever $f \prec g$ there is an $h \in C(X, \prec)$ such that $f$ $\leqslant h \leqslant g$.

The relationship between inclusions and lattice cones is given by the

Characterization Theorem. Let $X$ be a set, $\prec$ an idempotent inclusion on $X$, and let $K$ be a lattice cone on $X$.

(i) $C(X, \prec)$ is a closed lattice cone on $X$, and for $A, B \subset X, A \prec B$ iff there is an $f \in C(X, \prec)$ such that $1_{A} \leqslant f \leqslant 1_{B}$.

(ii) The binary relation $\prec_{K}$ on $2^{X}$ defined by

$$
A \prec_{K} B \Longleftrightarrow \exists f \in K \ni 1_{A} \leqslant f \leqslant 1_{B},
$$

is an idempotent inclusion on $X$ and $C\left(X, \prec_{K}\right)=\mathrm{cl}(K)$, the closure of $K$.

The Interposition and Characterization Theorems were announced in [3]. They will be proved in $\$ 2$. We shall make a number of applications of these theorems. The first of these applications will be interposition theorems and are to be found in $\S 3$. In this Introduction we limit ourselves to a single example. First, we need the notion of the product of two inclusions. For $\prec_{1}$ and $\prec_{2}$ inclusions on a set $X$, the product of $\prec_{1}$ and $\prec_{2}$ is the binary relation $\prec_{1} \times \prec_{2}$ on $2^{X}$ defined by

$$
A \prec_{1} \times \prec_{2} B \Leftrightarrow \exists C \subset X \ni A \prec_{1} C \text { and } C \prec_{2} B .
$$

Note that the product of two inclusions is again an inclusion and that an inclusion $\prec$ is idempotent iff $\prec x \prec=\prec-$ thus the term. Now let $K, L$ and $M$ be closed lattice cones on a set $X$. Then $K$ interposes $(L, M)$ iff $\prec_{L} \times \prec_{M} \subset \prec_{K}$. In case $K=L \cap M$, the condition " $\prec_{L} \times \prec_{M} \subset \prec_{K}$ " can be replaced by " $\prec_{L} \times$ $\prec_{M}$ is idempotent". As a special case we have the following 
THEOREM. Let $X$ be a topological space, $K$ the set of bounded continuous functions on $X, L$ the set of upper semicontinuous functions on $X$, and $M$ the set of lower semicontinuous functions on $X$.

(i) (Tong-Katětov [23], [24], [10], [11]) $K$ interposes $(L, M)$ iff $X$ is normal.

(ii) (Stone [22]) $K$ interposes $(M, L)$ iff $X$ is extremally disconnected.

In $\S 4$ we use the Characterization Theorem plus some ideas from functional analysis to prove Császár's compactification theorem [6, Chapter 16] for quasi-proximity spaces. A quasi-proximity $\operatorname{space}\left({ }^{4}\right)$ is a pair $(X, \delta)$ consisting of a set $X$ and a quasi-proximity $\delta$ on $X$, that is, a binary relation on $2^{X}$ which satisfies:

$\left(\mathrm{Q}_{1}\right)$ For $A \subset X, \varnothing \bar{\delta} A$ and $A \bar{\delta} \varnothing(\bar{\delta}$ denotes the negation of $\delta)$.

$\left(\mathrm{Q}_{2}\right) A \cap B \neq \varnothing \Rightarrow A \delta B$.

$\left(\mathrm{Q}_{3}\right) A \cup B \delta C \Longleftrightarrow A \delta C$ or $B \delta C$.

$\left(\mathrm{Q}_{4}\right) C \delta A \cup B \Longleftrightarrow C \delta A$ or $C \delta B$.

$\left(\mathrm{Q}_{5}\right) A \bar{\delta} B \Rightarrow \exists C \subset X \ni A \bar{\delta} C$ and $(X \sim C) \bar{\delta} B$.

The notions of quasi-proximity and idempotent inclusion are equivalent in that if $\delta$ (resp. $\prec$ ) is a quasi-proximity (resp. idempotent inclusion) on $X$, then the relation $\prec$ (resp. $\delta$ ) on $2^{X}$ defined by

$$
A \prec B \Longleftrightarrow A \bar{\delta}(X \sim B)
$$

is an idempotent inclusion (resp. quasi-proximity) on $X$. For $\delta$ a quasi-proximity on $X$, we define $C(X, \delta)$ to be $C(X, \prec), \prec$ the idempotent inclusion defined by $\delta$ via (IQ). Also via (IQ) we shall speak of the quasi-proximity defined by a lattice cone $K$ on $X$ : the quasi-proximity corresponding to the idempotent inclusion $\prec_{K}$ defined in the Characterization Theorem.

Now let $(X, \delta)$ be a quasi-proximity space. $(X, \delta)$ has a natural topologyas it turns out, the smallest topology on $X$ which renders continuous the members of $C(X, \delta)$-which we denote by $\tau(\delta)$, and all topological notions concerning $(X, \delta)$ refer to this topology. A compactification of $(X, \delta)$ is a pair $((Y, \eta), \kappa)$ consisting of a compact Hausdorff quasi-proximity space $(Y, \eta)$ and a mapping $\kappa: X \rightarrow Y$ such that
- $\kappa$ is a quasi-proximity embedding, i.e. $A \delta B$ iff $\kappa[A] \eta \kappa[B]$.
- $\kappa[X]$ is dense in $Y$.

The Császár Compactification Theorem states that $(X, \delta)$ has a compactification and, up to equivalence (defined in $\S 4$ ), only one.

(4) Quasi-proximity spaces were introduced by Pervin [17]. Pervin's definition was not quite correct in that Proposition 4.5 does not follow from his definition. Steiner [21] filled the gap in Pervin's definition and proved 4.5. 
In $\$ 5$ we use the Császar Compactification Theorem to describe the order compactifications and Nachbin compactifications of Nachbin's completely regular ordered topological spaces [15]. Since quite a number of definitions are required before these compactification theorems can be stated, we limit our discussion here to the remark that they are modelled on and carry as a special case Smirnov's description, in terms of certain proximities $\left({ }^{5}\right)$, of the topological compactifications of a completely regular topological space [19], [20].

In $\S 6$ we conclude the paper with an approximation theorem which extends a theorem of Nachbin [15, Appendix, §5, Theorem 6]. Namely, if $X$ is a set, $X_{0}$ a subset of $X, K$ a closed lattice cone on $X$, and if $K_{0}=\{k \in K: k$ is 0 on $\left.X_{0}\right\}$, then for any $f \in B(X)$, there is a $k_{0} \in K_{0}$ such that $\left\|f-k_{0}\right\|=$ $\inf \left\{\|f-k\|: k \in K_{0}\right\}$. Nachbin proved this theorem for the case that $X$ is a compact Hausdorff space, $X_{0}=\varnothing$, and $B(X)$ is replaced by $C(X)$. If in our setup one takes $X_{0}$ to be $\varnothing$, then our result follows easily from Nachbin's. Nachbin also gave a formula for $\inf \left\{\|f-k\|: k \in K_{0}\right\}$. We shall give a distance formula in terms of the quasi-proximity defined by $K$ and show that in Nachbin's set-up our formula reduces to his.

2. Proofs of the interposition and characterization theorems. We collect all the elementary facts about inclusions that we shall need in the following

2.1. Proposition. Let $X$ be $a$ set and let $\prec$ be an inclusion on $X$.

(i) $A \subset B<C \subset D \Rightarrow A<D$.

(ii) $A_{1} \prec B_{1}, A_{2} \prec B_{2} \Rightarrow A_{1} \cap A_{2} \prec B_{1} \cap B_{2}$ and $A_{1} \cup A_{2} \prec B_{1} \cup B_{2}$.

(iii) (For $f, g, h, k \in B(X)$ ), $f \leqslant g<h \leqslant k \Rightarrow f<k$.

(iv) $f \vee g<h \Longleftrightarrow f<h$ and $g<h$.

(v) $h \prec f \wedge g \Longleftrightarrow h \prec f$ and $h<g$.

(vi) $f_{1} \prec g_{1}, f_{2} \prec g_{2} \Rightarrow f_{1} \wedge f_{2} \prec g_{1} \wedge g_{2}$ and $f_{1} \vee f_{2} \prec g_{1} \vee g_{2}$.

(vii) $f_{1} \prec g_{1}, f_{2} \prec g_{2} \Rightarrow f_{1}+f_{2} \prec g_{1}+g_{2}$.

(viii) $f<g, 0 \leqslant \alpha \in \mathbf{R} \Rightarrow \alpha f<\alpha g$.

(ix) If $\left\{f_{n}\right\}_{n \in \mathrm{N}}$ and $\left\{g_{n}\right\}_{n \in \mathrm{N}}$ are sequences in $B(X)$ which converge, respectively, to $f$ and $g$, and if $f_{n} \prec g_{n}$ for all $n \in \mathrm{N}$, then $f \prec g$.

(x) If $0 \leqslant f \in B(X), 0 \leqslant t \in \mathbf{R}$, and if $A<\{x: f(x) \geqslant t\}$, then $t 1_{A}<f$.

Proof. (i) $A \cup B=B<C$ and so by $\left(\mathrm{I}_{3}\right) A \prec C$. $A \prec C=C \cap D$ and so by $\left(\mathrm{I}_{4}\right) A \prec D$.

(ii) By (i) $A_{1} \cap A_{2} \prec B_{1}$ and $A_{1} \cap A_{2} \prec B_{2}$. By (I $\left.A_{4}\right) A_{1} \cap A_{2} \prec B_{1}$ $\cap B_{2}$. Likewise one sees that $A_{1} \cup A_{2} \prec B_{1} \cup B_{2}$.

(5) A proximity is a symmetric quasi-proximity, i.e. $A \delta B \Rightarrow B \delta A$. This definition of proximity is due to Efremovič $[8],[9]$ although the general idea dates back to $F$. Riesz' $[18]$ address to the 1908 International Congress of Mathematicians. 
(iii), (iv), (v) and (vi) follow, respectively, from (i), $\left(\mathrm{I}_{3}\right),\left(\mathrm{I}_{4}\right)$ and (ii).

(vii) Let $r, s \in \mathrm{R}, r<s$. Set $\epsilon=(s-r) / 3$. We assert that

(1) $\left\{x:\left(f_{1}+f_{2}\right)(x) \geqslant s\right\} \subset \bigcup_{n \in Z}\left\{x: f_{1}(x) \geqslant s-n \epsilon\right\} \cap\left\{x: f_{2}(x) \geqslant(n-1) \epsilon\right\}$.

Let $\left(f_{1}+f_{2}\right)(x) \geqslant s$. Let $n \in \mathbf{Z}$ be such that $s-(n-1) \epsilon>f_{1}(x) \geqslant s-n \epsilon$. $f_{2}(x) \geqslant s-f_{1}(x)>s-(s-(n-1) \epsilon)=(n-1) \epsilon$. Thus (1) holds. For $n \in \mathbf{Z}$,

$$
\begin{aligned}
\left\{x: f_{1}(x) \geqslant s-n \epsilon\right\} & \prec\left\{x: g_{1}(x) \geqslant s-(n+1) \epsilon\right\}, \\
\left\{x: f_{2}(x) \geqslant(n-1) \epsilon\right\} & \prec\left\{x: g_{2}(x) \geqslant(n-2) \epsilon\right\}
\end{aligned}
$$

and so by (ii) we have

$$
\begin{aligned}
\left\{x: f_{1}(x)\right. & \geqslant s-n \epsilon\} \cap\left\{x: f_{2}(x) \geqslant(n-1) \epsilon\right\} \\
& \prec\left\{x: g_{1}(x) \geqslant s-(n+1) \epsilon\right\} \cap\left\{x: g_{2}(x) \geqslant(n-2) \epsilon\right\} .
\end{aligned}
$$

Clearly,

$\left\{x: g_{1}(x) \geqslant s-(n+1) \epsilon\right\} \cap\left\{x: g_{2}(x) \geqslant(n-2) \epsilon\right\} \subset\left\{x:\left(g_{1}+g_{2}\right)(x) \geqslant s-3 \epsilon\right\}$

$$
=\left\{x:\left(g_{1}+g_{2}\right)(x) \geqslant r\right\}
$$

and so by (i) and (2) we have

$$
\left\{x: f_{1}(x) \geqslant s-n \epsilon\right\} \cap\left\{x: f_{2}(x) \geqslant(n-1) \epsilon\right\} \prec\left\{x:\left(g_{1}+g_{2}\right)(x) \geqslant r\right\} .
$$

Since $f_{1}$ and $f_{2}$ are bounded, all but a finite number of the sets which appear in the union in (1) are empty. By $\left(\mathrm{I}_{3}\right)$ (plus a simple induction)

$$
\bigcup_{n \in \mathbf{Z}}\left\{x: f_{1}(x) \geqslant s-n \epsilon\right\} \cap\left\{x: f_{2}(x) \geqslant(n-1) \epsilon\right\}<\left\{x:\left(g_{1}+g_{2}\right)(x) \geqslant r\right\} .
$$

By (1) and (i) $\left\{x:\left(f_{1}+f_{2}\right)(x) \geqslant s\right\} \prec\left\{x:\left(g_{1}+g_{2}\right)(x) \geqslant r\right\}$.

(viii) is trivial.

(ix) Let $r, s \in \mathbf{R}, r<s$. Set $\epsilon=(s-r) / 3$. Let $n \in \mathrm{N}$ be such that $\left\|f-f_{n}\right\|,\left\|g-g_{n}\right\| \leqslant \epsilon$.

$$
\begin{aligned}
\{x: f(x) \geqslant s\} & \subset\left\{x: f_{n}(x) \geqslant s-\epsilon\right\} \\
\subset\{x: g(x) \geqslant s-3 \epsilon\} & =\{x: g(x) \geqslant r\} .
\end{aligned}
$$

(x) Set $B=\{x: f(x) \geqslant t\}$. By hypothesis $1_{A} \prec 1_{B}$ and so by (viii) $t 1_{A}$ $\prec t 1_{B}$. Since $t 1_{B} \leqslant f$, it follows from (iii) that $t 1_{A} \prec f$.

2.2. Proof of the Inter position Theorem. We first dispose of the simple implication "(b) $\Rightarrow$ (a)". Let $A, B \subset X, A \prec B .1_{A} \prec 1_{B}$ so that by (b) there is an $f \in C(X, \prec)$ such that $1_{A} \leqslant f \leqslant 1_{B}$. Set $C=\{x: f(x) \geqslant 1 / 2\} . A \subset$ $\{x: f(x) \geqslant 3 / 4\} \prec C \prec\{x: f(x) \geqslant 1 / 4\} \subset B$ and so by 2.1 (i) $A \prec C \prec B$. 
Now suppose (a) holds. Let $f, g \in B(X), f<g$. If $\alpha, \beta \in \mathbf{R}, \alpha>0$, and if $h \in C(X, \prec)$ is such that $\alpha(f+\beta) \leqslant h \leqslant \alpha(g+\beta)$, then $h / \alpha-\beta \in C(X, \prec)$ and $f \leqslant h / \alpha-\beta \leqslant g$. Thus we may assume that $f \geqslant 0$ and that $g(x)<1$ for all $x \in$ $X$. Suppose for the moment that we have constructed a sequence $\left\{h_{n}\right\}_{n \in \mathbb{N}}$ in $B(X)$ such that for each $n \in \mathrm{N}$,

(1) $0 \leqslant h_{n} \leqslant 1$,

(2) $h_{n}<g+2^{-n}$,

(3) $\left\{x: h_{n}(x) \geqslant(k+1) / 2^{n}\right\} \prec\left\{x: h_{n}(x) \geqslant k / 2^{n}\right\}$, all $k \in \mathbf{Z}$,

(4) $\left\{x: f(x) \geqslant(k+1) / 2^{n}\right\}<\left\{x: h_{n}(x) \geqslant k / 2^{n}\right\}$, all $k \in Z$,

(5) $h_{n}-3 \cdot 2^{-n-1} \leqslant h_{n+1} \prec h_{n}+3 \cdot 2^{-n-1}$.

By (5) $\left\{h_{n}\right\}_{n \in \mathrm{N}}$ converges, say to $h$, and by (5) and 2.1(ix) $h<h$. By (2) and 2.1(ix) $h \prec g$ and hence $h \leqslant g$. We assert that $h_{n}+2^{-n+1} \geqslant f$ for all $n$. Let $x \in X$. Let $k \in \mathbf{Z}$ be such that $k / 2^{n}>h_{n}(x) \geqslant(k-1) / 2^{n}$. By (4) $f(x)<$ $(k+1) / 2^{n} \leqslant h_{n}(x)+2^{-n+1}$. Thus $f \leqslant h \leqslant g$ and $h \in C(X, \prec)$.

We now construct the sequence $\left\{h_{n}\right\}_{n \in \mathbb{N}}$. Set $h_{1}=0$. (1)-(4) hold for $n=1$. Suppose $h_{1}, \ldots, h_{m}$ have been chosen such that (1)-(4) hold for $1 \leqslant$ $n \leqslant m$ and (5) for $1 \leqslant n<m$. For $k \in \mathbf{Z}$, set

$$
\begin{aligned}
& A_{k}=\left\{x: f(x) \geqslant(k+1) / 2^{m+1}\right\} \cup\left\{x: h_{m}(x) \geqslant(k+2) / 2^{m+1}\right\}, \\
& B_{k}=\left\{x: g(x) \geqslant(k-1) / 2^{m+1}\right\} \cap\left\{x: h_{m}(x) \geqslant(k-3) / 2^{m+1}\right\} .
\end{aligned}
$$

The first step in the construction of $h_{m+1}$ is to show that

$$
A_{k} \prec B_{k} \text {. }
$$

Since $f \prec g$,

$$
\left\{x: f(x) \geqslant(k+1) / 2^{m+1}\right\}<\left\{x: g(x) \geqslant(k-1) / 2^{m+1}\right\},
$$

and since $h_{m} \prec g+2^{-m}$,

$$
\left\{x: h_{m}(x) \geqslant \frac{k+2}{2^{m+1}}\right\}<\left\{x:\left(g+2^{-m}\right)(x) \geqslant \frac{k+1}{2^{m+1}}\right\}=\left\{x: g(x) \geqslant \frac{k-1}{2^{m+1}}\right\} .
$$

By $\left(I_{3}\right)$

$$
A_{k} \prec\left\{x: g(x) \geqslant(k-1) / 2^{m+1}\right\} .
$$

Let $j \in \mathbf{Z}$ be such that $k-1 \leqslant 2 j \leqslant k$.

$$
\begin{aligned}
\{x: f(x) \geqslant & \left.(k+1) / 2^{m+1}\right\} \subset\left\{x: f(x) \geqslant j / 2^{m}\right\} \\
& <\left\{x: h_{m}(x) \geqslant(j-1) / 2^{m}\right\} \subset\left\{x: h_{m}(x) \geqslant(k-3) / 2^{m+1}\right\} . \\
\left\{x: h_{m}(x) \geqslant(k+2) / 2^{m+1}\right\} \subset\left\{x: h_{m}(x) \geqslant(j+1) / 2^{m}\right\} & \\
& <\left\{x: h_{m}(x) \geqslant j / 2^{m}\right\} \subset\left\{x: h_{m}(x) \geqslant(k-3) / 2^{m+1}\right\} .
\end{aligned}
$$


Again by $\left(\mathrm{I}_{3}\right)$

$$
A_{k} \prec\left\{x: h_{m}(x) \geqslant(k-3) / 2^{m+1}\right\} .
$$

We now obtain (6) from (7), (8) and $\left(\mathrm{I}_{4}\right)$. We next construct $C_{0}, \ldots, C_{2^{m+1}}$ $\subset X$ such that

$$
\begin{aligned}
& A_{k} \prec C_{k} \prec B_{k}, \quad k=0, \ldots, 2^{m+1}, \\
& C_{k} \prec C_{k-1}, \quad k=1, \ldots, 2^{m+1} .
\end{aligned}
$$

Since $\prec$ is idempotent, there is a $C_{0} \subset X$ such that $A_{0} \prec C_{0} \prec B_{0}$. Suppose $p$ $<2^{m+1}$ and $C_{0}, \ldots, C_{p}$ have been chosen in accordance with (9) and (10). $A_{p+1} \prec B_{p+1}$ and $A_{p+1} \subset A_{p} \prec C_{p}$ so that $A_{p+1} \prec C_{p} \cap B_{p+1}$. Thus there is a $C_{p+1} \subset X$ such that $A_{p+1} \prec C_{p+1} \prec C_{p} \cap B_{p+1}$. Thus we can find $C_{0}$, $\ldots, C_{2 m+1}$ which satisfy (9) and (10). Set

$$
h_{m+1}=\bigvee_{k=1}^{2 m+1} k 2^{-m-1} 1_{c_{k}} .
$$

Clearly, $h_{m+1}$ satisfies (1). For each $k, C_{k} \prec\left\{x:\left(g+2^{-m-1}\right)(x) \geqslant k / 2^{m+1}\right\}$ and so by $2.1(\mathrm{x}) k \cdot 2^{-m-1} 1_{C_{k}} \prec g+2^{-m-1}$. By $2.1(\mathrm{iv}), h_{m+1} \prec g+2^{-m-1}$, i.e. $h_{m+1}$ satisfies (2). For $k=1, \ldots, 2^{m+1},\left\{x: h_{m+1}(x) \geqslant k / 2^{m+1}\right\}=C_{k}$ (since the $C_{k}$ are decreasing) and so by (10) we have

$$
\begin{array}{r}
\left\{x: h_{m+1}(x) \geqslant(k+1) / 2^{m+1}\right\}<\left\{x: h_{m+1}(x) \geqslant k / 2^{m+1}\right\}, \\
k=1, \ldots, 2^{m+1}-1 .
\end{array}
$$

If $k \leqslant 0$, then $\left\{x: h_{m+1}(x) \geqslant k / 2^{m+1}\right\}=X$, and if $k \geqslant 2^{m+1}$, then $\{x$ : $\left.h_{m+1}(x) \geqslant(k+1) / 2^{m+1}\right\}=\varnothing$. Thus $h_{m+1}$ satisfies (3). By (9)

$$
\begin{aligned}
\left\{x: f(x) \geqslant(k+1) / 2^{m+1}\right\}<C_{k}=\left\{x: h_{m+1}(x) \geqslant k / 2^{m+1}\right\}, & \\
& k=1, \ldots, 2^{m+1} .
\end{aligned}
$$

If $k \leqslant 0$, then $\left\{x: h_{m+1}(x) \geqslant k / 2^{m+1}\right\}=X$, and if $k>2^{m+1}$, then $\{x: f(x)$ $\left.\geqslant(k+1) / 2^{m+1}\right\}=\varnothing$. Thus $h_{m+1}$ satisfies (4). By (9)

$$
C_{k} \prec\left\{x:\left(h_{m}+3 \cdot 2^{-m-1}\right)(x) \geqslant k / 2^{m+1}\right\}, \quad k=1, \ldots, 2^{m+1},
$$

and so by $2.1(\mathrm{x})$

$$
k \cdot 2^{-m-1} 1_{C_{k}} \prec h_{m}+3 \cdot 2^{-m-1}, \quad k=1, \ldots, 2^{m+1} .
$$

By 2.1(iv) $h_{m+1} \prec h_{m}+3 \cdot 2^{-m-1}$. Now let $x \in X$. Let $k \in \mathrm{N}$ be such that $k / 2^{m+1}>h_{m+1}(x) \geqslant(k-1) / 2^{m+1}$. If $k=2^{m+1}+1$, then 


$$
h_{m+1}(x)=1>g(x) \geqslant h_{m}(x)-2^{-m}>h_{m}(x)-3 \cdot 2^{-m-1} .
$$

If $k \leqslant 2^{m+1}$, then $\left\{y: h_{m+1}(y) \geqslant k / 2^{m+1}\right\}=C_{k}$ and so $x \notin C_{k}$. By (9), $h_{m}(x)$ $<(k+2) / 2^{m+1} \leqslant h_{m+1}(x)+3 \cdot 2^{-m-1}$. Thus $h_{m+1} \geqslant h_{m}-3 \cdot 2^{-m-1}$ and we are done.

2.3. Proof of the Characterization Theorem. (i) That $C(X, \prec)$ is a closed lattice cone on $X$ follows immediately from (vi)-(ix) of 2.1. Let $A, B$ $\subset X$. If $A \prec B$, then $1_{A} \prec 1_{B}$, and so by the Interposition Theorem there is an $f \in C(X, \prec)$ such that $1_{A} \leqslant f \leqslant 1_{B}$. If, on the other hand, $1_{A} \leqslant f \leqslant 1_{B}$ for some $f \in C(X, \prec)$, we have $1_{A} \leqslant f \prec f \leqslant 1_{B}$ and so by 2.1 (iii) $1_{A} \prec 1_{B}$, i.e. $A$ $<B$.

(ii) The relation $\prec_{K}$ is easily seen to be an inclusion on $X$. That it is idempotent will follow from the fact that $K \subset C\left(X, \prec_{K}\right)$. . Thus, let $f \in K, r, s$ $\in \mathbf{R}, r<s$. Set $g=1 \wedge[1 /(s-r)((f-r) \vee 0)] . g$ belongs to $K$, is 1 on $E=\{x: f(x)$ $\geqslant s\}, 0$ off $F=\{x: f(x) \geqslant r\}$, and $0 \leqslant g \leqslant 1$, i.e. $1_{E} \leqslant g \leqslant 1_{F}$, i.e. $E \prec_{K} F$. Therefore $f \in C\left(X, \prec_{K}\right)$. Now let $A \prec_{K} B$. Let $f \in K$ be such that $1_{A} \leqslant f \leqslant$ $1_{B}$. Set $C=\{x: f(x) \geqslant 1 / 2\}$.

$$
A \subset\{x: f(x) \geqslant 3 / 4\} \cdot \prec_{K} C \prec_{K}\{x: f(x) \geqslant 1 / 4\} \subset B
$$

and so $A \prec_{K} C \prec_{K} B$. Thus $\prec_{K}$ is idempotent.

Since $K \subset C\left(X, \prec_{K}\right)$ and since $C\left(X, \prec_{K}\right)$ is closed, we need only show that $C\left(X, \prec_{K}\right) \subset \operatorname{cl}(K)$. Since $C\left(X, \prec_{K}\right)$ and $\operatorname{cl}(K)$ contain the constants, it is enough to show that $\mathrm{cl}(K)$ contains the nonnegative members of $C\left(X, \prec_{K}\right)$. Let $0 \leqslant f \in$ $C\left(X, \prec_{K}\right)$. Let $\epsilon>0$, and let $N \in \mathrm{N}$ be such that $f \leqslant N \epsilon$. Set $E_{n}=\{x: f(x)$ $\geqslant n \epsilon\}, n=0, \ldots, N+1$. Since $f \in C\left(X, \prec_{K}\right), E_{n} \prec_{K} E_{n-1}, n=1, \ldots, N$

+1. Let $g_{n} \in K$ be such that $1_{E_{n}} \leqslant g_{n} \leqslant 1_{E_{n-1}}, n=1, \ldots, N+1 . f+\epsilon \geqslant$ $n \epsilon 1_{E_{n-1}} \geqslant n \in g_{n}, n=1, \ldots, N+1$, and so $f+\epsilon \geqslant g=\bigvee_{n=1}^{N+1} n \in g_{n}$. Let $x \in X$, and let $n$ be such that $(n+1) \epsilon>f(x) \geqslant n \epsilon . x \in E_{n}$ and so $g_{n}(x)=$ 1. $g(x) \geqslant n \epsilon g_{n}(x)=n \epsilon>(f-\epsilon)(x)$. Therefore $f-\epsilon \leqslant g \leqslant f+\epsilon$, i.e. $\|f-g\|$ $\leqslant \epsilon$.

3. Interposition by lattice cones of functions. In this section we shall extend a number of results which were proved or were to have been announced in [2] .

3.1. Definition. For $X$ a set and $K, L$ and $M$ subsets of $B(X)$, we shall say that

$K$ (simply) interposes the pair $(L, M)$ if whenever $f \in L, g \in M$ and $f \leqslant g$, there is an $h \in K$ such that $f \leqslant h \leqslant g$,

$K$ strictly interposes the pair $(L, M)$ if whenever $f \in L, g \in M$, and $f<g$ (i.e. $f(x)<g(x)$ for all $x \in X$ ), there is an $h \in K$ such that $f<h<g$,

$K$ ultrastrictly interposes the pair $(L, M)$ if whenever $f \in L, g \in M$, and 
$f \leqslant g$, there is an $h \in K$ such that $f \leqslant h \leqslant g$ and $f(x)<h(x)<g(x)$ if $f(x)<$ $g(x)$.

The notions of simple, strict, and ultrastrict interposition have their origins in the following results.

Theorem. Let $X$ be a topological space, $K$ the set of continuous functions on $X, L$ the upper semicontinuous functions on $X$, and $M$ the lower semicontinuous functions.

(i) (Katětov-Tong [23], [24], [10], [11]) $K$ interposes $(L, M)$ iff $X$ is normal.

(ii) (Dowker [7]) $K$ strictly interposes $(L, M)$ iff $X$ is normal and countably paracompact.

(iii) (Michael [14]) $K$ ultrastrictly interposes $(L, M)$ iff $X$ is perfectly normal.

Our interposition theorems are modelled on the above theorem, and (i) and (iii) are easy corollaries of our results. (ii) is obtained from our result on strict interposition via Dowker's characterization of countable paracompactness [7, Theorem 2].

We begin with some elementary facts about products of inclusions.

3.2. Proposition. Let $X$ be $a$ set and let $\prec_{1}$ and $\prec_{2}$ be inclusions on $X$.

(i) $\prec_{1} \times \prec_{2}$ is an inclusion on $X$.

(ii) If $f_{1} \in C\left(X, \prec_{1}\right), f_{2} \in C\left(X, \prec_{2}\right)$, and if $f_{1} \leqslant f_{2}$, then $f_{1} \prec_{1} \times \prec_{2} f_{2}$.

(iii) $C\left(X, \prec_{1} \times \prec_{2}\right)=C\left(X, \prec_{1}\right) \cap C\left(X, \prec_{2}\right)$.

PRoof. (i) is easily verified.

(ii) Let $r, s \in \mathbf{R}, r<s$.

$$
\begin{aligned}
\left\{x: f_{1}(x) \geqslant s\right\} & \prec_{1}\left\{x: f_{1}(x) \geqslant(r+s) / 2\right\} \subset\left\{x: f_{2}(x) \geqslant(r+s) / 2\right\} \\
& \prec_{2}\left\{x: f_{2}(x) \geqslant r\right\}
\end{aligned}
$$

and so $\left\{x: f_{1}(x) \geqslant s\right\} \prec_{1} x \prec_{2}\left\{x: f_{2}(x) \geqslant r\right\}$. Thus $f_{1} \prec_{1} \times \prec_{2} f_{2}$.

(iii) By (ii) $C\left(X, \prec_{1}\right) \cap C\left(X, \prec_{2}\right) \subset C\left(X, \prec_{1} \times \prec_{2}\right)$. Since $A \prec_{1} \times \prec_{2} B$ implies that $A \prec_{1} B$ and $A \prec_{2} B$, we have $C\left(X, \prec_{1} \times \prec_{2}\right) \subset C\left(X, \prec_{1}\right) \cap C\left(X, \prec_{2}\right)$.

3.3. TheOREM. Let $X$ be a set, and let $K, L$ and $M$ be closed lattice cones on $X$. Then $K$ interposes $(L, M)$ iff $\prec_{L} \times \prec_{M} \subset \prec_{K}$, i.e. $A \prec_{L} \times \prec_{M} B \Rightarrow A \prec_{K} B$.

Proof. (if). Let $f \in L, g \in M, f \leqslant g$. By the Characterization Theorem $L=C\left(X, \prec_{L}\right)$ and $M=C\left(X, \prec_{M}\right)$, and by (ii) of $3.2 f \prec_{L} \times \prec_{M} g$. By hypothesis $f \prec_{K} g$. By the Interposition and Characterization Theorems there is an $h \in K$ such that $f \leqslant h \leqslant g$.

(only if). Let $A \prec_{L} \times \prec_{M} B$. There is a $C \subset X$ such that $A \prec_{L} C \prec_{M} B$ 
and so there exist $f \in L$ and $g \in M$ such that $1_{A} \leqslant f \leqslant 1_{C} \leqslant g \leqslant 1_{B}, f \leqslant g$ and so there is an $h \in K$ such that $f \leqslant h \leqslant g .1_{A} \leqslant h \leqslant 1_{B}$ and so $A \prec_{K} B$.

In the case that $K=L \cap M$ we can sharpen 3.3 somewhat:

3.4. TheOREm. Let $X$ be a set, and let $L$ and $M$ be closed lattice cones on $X$. Then $L \cap M$ interposes $(L, M)$ iff $\prec_{L} \times \prec_{M}$ is idempotent.

Proof. Set $K=L \cap M$. Suppose $\prec_{L} \times \prec_{M}$ is idempotent. By 3.2(iii) and the Characterization Theorem we have $C\left(X, \prec_{L} \times \prec_{M}\right)=C\left(X, \prec_{L}\right) \cap C\left(X, \prec_{M}\right)$ $=K$, and so, once again by the Characterization Theorem, $\prec_{L} \times \prec_{M}=\prec_{K}$. By 3.3 $K$ interposes $(L, M)$. Now suppose $L \cap M$ interposes $(L, M)$. By $3.3 \prec_{L} \times \prec_{M}$ $\subset \prec_{L \cap M}$ and trivally $\prec_{L \cap M} \subset \prec_{L} \times \prec_{M}$. Thus $\prec_{L} \times \prec_{M}=\prec_{L \cap M}$ and so $\prec_{L} \times \prec_{M}$ is idempotent.

We next obtain as a corollary to 3.4 a result from [2]. We need some definitions.

3.5. Definition. Let $X$ be a set, and let $C$ be a sublattice of $2^{X}$ to which $\varnothing$ and $X$ belong. ${ }_{c}$ is the binary relation on $2^{X}$ defined by

$$
A \prec_{C} B \Longleftrightarrow \exists C \in C \ni A \subset C \subset B .
$$

The smallest convex cone in $B(X)$ which contains the constant functions and the characteristic functions $1_{C}, C \in C$, is denoted by $\mathrm{cn}(C)$ and its closure is denoted by $\overline{\operatorname{cn}}(\mathrm{C})$.

3.6. Proposition. Let $X$ be a set, and let $C$ be a sublattice of $2^{X}$ to which $\varnothing$ and $X$ belong.

(i) $\prec_{c}$ is an idempotent inclusion on $X$.

(ii) $C\left(X, \prec_{C}\right)=\overline{\operatorname{cn}}(C)$.

PROOF. (i) is easily verified.

(ii) $C\left(X, \prec_{C}\right)$ is a closed convex cone which contains the constant functions and the characteristic functions $1_{C}, C \in \mathcal{C}$, and so $C\left(X, \prec_{C}\right) \supset \overline{\mathrm{cn}}(C)$. Let $0 \leqslant f \in C\left(X, \prec_{c}\right)$, and let $\epsilon>0$. Let $N \in \mathrm{N}$ be such that $N \epsilon \geqslant f$. Let $C_{n} \in \mathcal{C}$ be such that $\{x: f(x) \geqslant n \epsilon\} \subset C_{n} \subset\{x: f(x) \geqslant(n-1) \epsilon\}, n=0, \ldots, N+1$. $f+\epsilon \geqslant n \epsilon 1_{C_{n}}, n=1, \ldots, N+1$, and so $f+\epsilon \geqslant g=\bigvee_{n=1}^{N+1} n \epsilon 1_{C_{n}}=$ $\Sigma_{n=1}^{N+1} \epsilon 1_{C_{n}} \in \mathrm{cn}(C)$. Let $x \in X$. Let $n \in \mathrm{N}$ be such that $n \epsilon>f(x) \geqslant(n-1) \epsilon$. $x \in C_{n-1}$ and so $g(x)+\epsilon \geqslant n \epsilon>f(x)$. Thus $g \geqslant f-\epsilon$. It follows that $\|f-g\|$ $\leqslant \epsilon$.

3.7. Proposition. Let $X$ be $a$ set and let $A$ and $B$ be sublattices of $2^{X}$ to which $\varnothing$ and $X$ belong. Then $\prec_{A} \times \prec_{B}$ is idempotent iff whenever $A \in A, B \in B$, and $A \subset B$, there exist $A^{\prime} \in A$ and $B^{\prime} \in B$ such that $A \subset B^{\prime} \subset A^{\prime} \subset B$. 
Proof. Straightforward.

The 'if' part of the following result was proved in [2], and the 'only if' part was to have been announced there.

3.8. THEOREM. Let $X$ be $a$ set and let $A$ and $B$ be sublattices of $2^{X}$ to which $\varnothing$ and $X$ belong. Then $\overline{\operatorname{cn}}(A) \cap \overline{\operatorname{cn}}(B)$ interposes $(\overline{\operatorname{cn}}(A), \overline{\operatorname{cn}}(B))$ iff whenever $A \in A, B \in B$ and $A \subset B$, there exist $A^{\prime} \in A, B^{\prime} \in B$ such that $A \subset B^{\prime} \subset$ $A^{\prime} \subset B$.

Proof. By 3.6 and the Characterization Theorem $\prec_{A}=\prec_{\overline{c n}(A)}$ and $\prec_{B}=$ $\prec_{\overline{c n}(B)}$. The theorem now follows from 3.4 and 3.7.

We now take up the matter of strict interposition. Although in general the strict interposition of $(L, M)$ by $K$ does not imply interposition, we do have this implication in the case that $K, L$ and $M$ are closed lattice cones and $K=L \cap M$.

3.9. Proposition. Let $X$ be a set and let $L$ and $M$ be closed lattice cones on $X$ such that $L \cap M$ strictly interposes $(L, M)$. Then $L \cap M$ interposes $(L, M)$.

PROoF. Let $f \in L, g \in M, f \leqslant g$. Let $h_{1} \in L \cap M$ be such that $f-1 / 2<$ $h_{1}<g$. Suppose $h_{1}, \ldots, h_{n} \in L \cap M$ have been chosen such that

(1) $f-2^{-k}<h_{k}<g, k=1, \ldots, n$,

(2) $h_{k}<h_{k+1}<h_{k}+2^{-k-1}, k=1, \ldots, n-1$. $\left(f-2^{-n-1}\right) \vee h_{n}<g \vee\left(h_{n}+2^{-n-1}\right)$ and so there is an $h_{n+1} \in L \cap M$ such that $\left(f-2^{-n-1}\right) \vee h_{n}<h_{n+1}<g \vee\left(h_{n}+2^{-n-1}\right)$. Thus we can find a sequence $\left\{h_{n}\right\}_{n \in \mathbf{N}}$ in $L \cap M$ such that (1) and (2) hold for all $n \in \mathbf{N}$. By (2) $\left\{h_{n}\right\}_{n \in \mathbf{N}}$ converges, say to $h$, and by (1) $f \leqslant h \leqslant g$.

3.10. Definition. Let $X$ be a set and let $K$ be a lattice cone on $X$. We shall say that a sequence $\left\{A_{n}\right\}_{n \in \mathrm{N}}$ in $2^{X} K$-increases (resp. $K$-decreases) to $A \subset$ $X$ if $\bigcup_{n \in \mathrm{N}} A_{n}=A$ (resp. $\bigcap_{n \in \mathrm{N}} A_{n}=A$ ) and for all $n \in N, A_{n} \prec_{K} A_{n+1}$ (resp. $\left.A_{n+1} \prec_{K} A_{n}\right)$.

3.11. THEOREM. Let $X$ be a set and let $L$ and $M$ be closed lattice cones on $X$ such that either $-L \subset M$ or $-M \subset L$. Then $L \cap M$ strictly interposes $(L, M)$ iff:

(1) $L \cap M$ interposes $(L, M)$.

(2) Whenever $\left\{A_{n}\right\}_{n \in \mathrm{N}}$ is a sequence in $2^{X}$ which L-decreases to $\varnothing$, there is a sequence $\left\{B_{n}\right\}_{n \in \mathrm{N}}$ in $2^{X}$ which M-decreases to $\varnothing$ and $B_{n} \supset A_{n}$ for all $n \in$ N.

(3) Whenever $\left\{A_{n}\right\}_{n \in N}$ is a sequence in $2^{X}$ which M-increases to $X$, there is a sequence $\left\{B_{n}\right\}_{n \in \mathrm{N}}$ in $2^{X}$ which L-increases to $X$ and $B_{n} \subset A_{n}$ for all $n \in \mathbf{N}$.

Proof. We first consider the case that $-L \subset M$. Suppose (1), (2) and (3) hold, and suppose for the moment we have proved 
(4) $0<g \in M \Rightarrow \exists f \in L \ni 0<f \leqslant g$.

Let $f \in L, g \in M$, and $f<g . \quad 0<g-f \in M$ and so by (4) there is an $h \in L$ such that $0<h \leqslant g-f$. $f+h / 2 \in L, g-h / 2 \in M$, and $f+h / 2 \leqslant g-h / 2$. By (1) there is a $k \in L \cap M$ such that $f+h / 2 \leqslant k \leqslant g-h / 2$. Since $h>0, f<k$ $<g$. Thus it is enough to prove (4). Let $0<g \in M$. For $n \in \mathrm{N}$, set $A_{n}=\{x$ : $\left.g(x) \geqslant 2^{-n+1}\right\}$. Since $g>0, X=\bigcup_{n \in \mathrm{N}} A_{n}$, and since $g \in M, A_{n} \prec_{M} A_{n+1}$ for all $n \in \mathrm{N}$. By (3) there is a sequence $\left\{B_{n}\right\}_{n \in \mathrm{N}}$ in $2^{X}$ which $L$-increases to $X$ and $A_{n} \prec B_{n}$ for all $n \in N$. Set $B_{0}=\varnothing$, and let $f_{n} \in L$ be such that $1_{B_{n-1}}$ $\leqslant f_{n} \leqslant 1_{B_{n}}$. Set $f=\Sigma_{n \in N^{2}} 2^{-n} f_{n}$. Clearly, $f>0$ and $f \in L$. Let $x \in X$. If $g(x) \geqslant 1$, then certainly $g(x) \geqslant f(x)$. Let $g(x)<1$. Let $n \in \mathrm{N}$ be such that $2^{-n+1}>g(x) \geqslant 2^{-n}$. $f(x)=\Sigma_{k>n} 2^{-k} f_{k}(x) \leqslant \Sigma_{k>n} 2^{-k}=2^{-n} \leqslant g(x)$. Thus (4) holds and so $L \cap M$ strictly interposes $(L, M)$.

Now suppose $L \cap M$ strictly interposes $(L, M)$. By 3.9 (1) holds. Let $\left\{A_{n}\right\}_{n \in \mathrm{N}} L$-decrease to $\varnothing$. For $n \in \mathrm{N}$, let $f_{n} \in L$ be such that $1_{A_{n+1}} \leqslant f_{n} \leqslant$ $1_{A_{n}}$. Set $f=\Sigma_{n \in \mathrm{N}} 2^{-n} f_{n} . f \in L$ and $f<1$. Thus there is a $g \in L \cap M$ such that $f<g<1$. Let $B_{n}=\left\{x: g(x) \geqslant 1-2^{-n+1}\right\}$. Clearly, $\left\{B_{n}\right\}_{n \in \mathrm{N}} M$-decreases to $\varnothing, B_{1}=X$ and for $n \geqslant 2$, if $x \in A_{n}, g(x)>f(x) \geqslant \sum_{k=1}^{n-1} 2^{-k}=1-$ $2^{-n+1}$ and so $x \in B_{n}$. Thus (2) holds. Now let $\left\{A_{n}\right\}_{n \in \mathrm{N}} M$-increase to $X$. For $n \in \mathrm{N}$, let $g_{n} \in M$ be such that $1_{A_{n}} \leqslant g_{n} \leqslant 1_{A_{n+1}}$. Set $g=\Sigma_{n \in \mathrm{N}} 2^{-n} g_{n}$. $0<g \in M$ and so there is an $f \in L \cap M$ such that $0<f<g$. Set $B_{n}=\{x: f(x)$ $\left.\geqslant 2^{-n+1}\right\} . \quad\left\{B_{n}\right\}_{n \in \mathbb{N}} L$-increases to $X$. If $x \in X \sim A_{n}$, then $f(x)<g(x)=$ $\Sigma_{k \geqslant n} 2^{-k} g_{k}(x) \leqslant \Sigma_{k \geqslant n} 2^{-k}=2^{-n+1}$ and so $x \notin B_{n}$. Thus $B_{n} \subset A_{n}$ and so (3) holds.

Thus if we are given that $-L \subset M, L \cap M$ strictly interposes $(L, M)$ iff (1), (2) and (3) hold. Now let $-M \subset L$. Note that $-(-M) \subset-L$ and that $L \cap M$ (strictly) interposes $(L, M)$ iff $(-M) \cap(-L)$ (strictly) interposes $(-M,-L)$. Thus we shall be done once we have shown that the pair $(-M,-L)$ satisfies (2) and (3) iff $(L, M)$ does. If $K$ is a lattice cone on $X$, then a sequence $\left\{A_{n}\right\}_{n \in \mathrm{N}}$ in $2^{X}$ $K$-increases to $X$ (resp. $K$-decreases to $\varnothing$ ) iff $\left\{X \sim A_{n}\right\}_{n \in \mathrm{N}}(-K)$-decreases to $\varnothing$ (resp. $(-K)$-increases to $X)$. Thus $(L, M)$ satisfies (2) (resp. (3)) iff $(-M,-L)$ satisfies (3) (resp. (2)).

In the special case that $L=\overline{\operatorname{cn}}(A)$ and $M=\overline{\operatorname{cn}}(B), 3.11$ takes the following form.

3.12. THEOREM. Let $X$ be a set and let $A$ and $B$ be sublattices of $2^{X}$ to which $\varnothing$ and $X$ belong. Suppose also that either $B \supset A^{c}(=\{X \sim A: A \in A\})$ or $A \supset B^{c}$. Then $\overline{\operatorname{cn}}(A) \cap \overline{c n}(B)$ strictly interposes $(\overline{\operatorname{cn}}(A), \overline{c n}(B))$ iff:

(1) $\overline{\mathrm{cn}}(A) \cap \overline{\mathrm{cn}}(B)$ interposes $(\overline{\mathrm{cn}}(A), \overline{\mathrm{cn}}(B))$.

(2) Whenever $\left\{A_{n}\right\}_{n \in \mathrm{N}}$ is a sequence in $A$ which decreases to $\varnothing$, there is a sequence $\left\{B_{n}\right\}_{n \in \mathrm{N}}$ in $B$ which decreases to $\varnothing$ and $B_{n} \supset A_{n}$ for all $n \in \mathbf{N}$. 
(3) Whenever $\left\{B_{n}\right\}_{n \in \mathrm{N}}$ is a sequence in $B$ which increases to $X$, there is a sequence $\left\{A_{n}\right\}_{n \in \mathrm{N}}$ in $\mathrm{A}$ which increases to $X$ and $A_{n} \subset B_{n}$ for all $n \in \mathrm{N}$.

Proof. Set $L=\overline{\operatorname{cn}}(A), M=\overline{\operatorname{cn}}(B) . \overline{\operatorname{cn}}\left(A^{c}\right)=-L$ and $\overline{\operatorname{cn}}\left(B^{c}\right)=-M$ so that either $-L \subset M$ or $-M \subset L$. We must verify that $L$ and $M$ satisfy (1), (2) and (3) of 3.11 iff $A$ and $B$ satisfy (1), (2) and (3). (1) and 3.11(1) are the same. Suppose (1), (2) and (3) hold. Let $\left\{A_{n}\right\}_{n \in N} L$-decrease to $\varnothing$. $\prec_{L}=\prec_{A}$ and so for each $n \in \mathrm{N}$ there is an $A_{n}^{\prime} \in \mathrm{A}$ such that $A_{n+1} \subset A_{n}^{\prime} \subset A_{n}$. By (2) we have a sequence $\left\{B_{n}^{\prime}\right\}_{n \in \mathrm{N}}$ in $B$ which decreases to $\varnothing$ and for each $n \in \mathrm{N}$, $B_{n}^{\prime} \supset A_{n}^{\prime}$. Set $B_{1}=X$, and for $n>1$, set $B_{n}=B_{n-1}^{\prime} \cdot\left\{B_{n}\right\}_{n \in \mathrm{N}} M$-decreases to $\varnothing$ and $A_{n} \subset B_{n}$ for all $n \in \mathbf{N}$. Thus 3.11(2) holds. Similarly, 3.11(3) is seen to hold. Now let (1), (2) and (3) of 3.11 hold. Let $\left\{A_{n}\right\}_{n \in N}$ be a sequence in A which decreases to $\varnothing$. $\left\{A_{n}\right\}_{n \in \mathrm{N}} L$-decreases to $\varnothing$ and so there is a sequence $\left\{B_{n}^{\prime}\right\}_{n \in \mathrm{N}}$ in $2^{X}$ which $M$-decreases to $\varnothing$ and for all $n \in \mathrm{N}, B_{n}^{\prime} \supset A_{n}$. Let $B_{n}^{\prime \prime} \in$ $B$ be such that $B_{n+1}^{\prime} \subset B_{n}^{\prime \prime} \subset B_{n}^{\prime}$. Set $B_{1}=X$, and for $n>1$, set $B_{n}=B_{n-1}^{\prime \prime}$. Likewise one sees that (3) holds.

It would be nice if one could drop the hypothesis " $-L \subset M$ or $-M \subset L$ " in 3.11, and, to a lesser extent, it would also be nice if one could replace $L \cap M$ by an arbitrary cone. The 'if' part of 3.12 was proved in [2] and the 'only if' part was to have been announced there.

We now take up ultrastrict interposition. First, some preparation.

3.13. Definition. Let $X$ be a set. For $f \in B(X), N(f)=\{x: f(x) \geqslant 0\}$, and $P(f)=\{x: f(x)>0\}$. For $K \subset B(X), N(K)=\{N(f): f \in K\}$ and $P(K)=$ $\{P(f): f \in K\}$.

3.14. Lemma. Let $X$ be a set and let $K$ be a closed lattice cone on $X$.

(i) $P(K)$ and $N(K)$ are sublattices of $2^{X}$ to which $\varnothing$ and $X$ belong.

(ii) $P(K)$ (resp. $N(K)$ ) is closed under countable unions (resp. intersections).

(iii) $\overline{\mathrm{cn}}(P(K))$ (resp. $\overline{\mathrm{cn}}(N(K))$ ) consists of those $f \in B(X)$ for which there is a sequence in $K$ which increases (resp. decreases) to $f$.

Proof. The statements about $N(K)$ follow from the corresponding statements about $P(K)$ and the observation that $N(K)=\{X \sim A: A \in P(-K)\}$. Thus we shall prove only the statements about $P(K)$.

We prove (i) and (ii) together. For $f, g \in K, P(f) \cap P(g)=P(f \wedge g)$. Let $\left\{f_{n}\right\}_{n \in \mathrm{N}}$ be a sequence in $K \sim\{0\} . P\left(\Sigma_{n \in \mathrm{N}} 2^{-n}\left\|f_{n}\right\|^{-1}\left(f_{n} \vee 0\right)\right)=\bigcup_{n \in \mathrm{N}} P\left(f_{n}\right)$. $\varnothing=P(0), X=P(1)$.

(iii) The set $L$ of those $f \in B(X)$ for which there is a sequence in $K$ which increases pointwise to $f$ is easily seen to be a lattice cone on $X$. Let $f \in \operatorname{cl}(L)$. Let $\left\{f_{n}\right\}_{n \in \mathrm{N}}$ be an increasing sequence in $L$ which converges to $f$, and for each $n \in \mathrm{N}$, let $\left\{f_{n, k}\right\}_{k \in \mathrm{N}}$ be a sequence in $K$ which increases to $f_{n}$. Set $g_{n}=\bigvee_{i, j=1}^{n} f_{i, j}$. 
Then $\left\{g_{n}\right\}_{n \in \mathbb{N}}$ increases to $f$. Thus $L$ is a closed lattice cone on $X$. Let $C \in$ $P(K)$, say $C=P(f)$. Since $P(f)=P(f \vee 0)$ we may assume that $f \geqslant 0$. $\{1 \wedge$ $n f\}_{n \in \mathrm{N}}$ increases pointwise to $1_{C}$ and so $1_{C} \in L$. Thus $L \supset \overline{\mathrm{cn}}(P(K))$. Now let $f \in L$. Let $\left\{f_{n}\right\}_{n \in \mathbf{N}}$ be a sequence in $K$ which increases to $f$. Let $r, s \in \mathbf{R}, r<$ s.

$$
\{x: f(x)>(r+s) / 2\}=\bigcup_{n \in \mathbb{N}}\left\{x: f_{n}(x)>(r+s) / 2\right\} \in P(K)
$$

and

$$
\{x: f(x) \geqslant s\} \subset\{x: f(x)>(r+s) / 2\} \subset\{x: f(x) \geqslant r\} .
$$

Thus $f \in C\left(X, \prec_{P(K)}\right)=\overline{c n}(P(K))$.

3.15. Theorem. Let $X$ be $a$ set and let $K, L$ and $M$ be closed lattice cones on $X$. Then $K$ ultrastrictly interposes $(L, M)$ iff

(1) $K$ interposes $(L, M)$,

(2) $\prec_{L} \subset \prec_{N(K)}$ and $\prec_{M} \subset \prec_{P(K)}$.

Proof. (if). Let $f \in L, g \in M, f \leqslant g . \quad L \subset \overline{\operatorname{cn}}(N(K))$ and $M \subset \overline{\operatorname{cn}}(P(K))$ so that by 3.14(iii) we can find sequences $\left\{f_{n}\right\}_{n \in \mathrm{N}}$ and $\left\{g_{n}\right\}_{n \in \mathrm{N}}$ in $K$ which, respectively, decrease to $f$ and increase to $g$. Let $h \in K$ be such that $f \leqslant h \leqslant g$. Set $k=\Sigma_{n \in \mathrm{N}} 2^{-n-1}\left(f_{n} \wedge h+g_{n} \vee h\right) . f \leqslant\left(f_{n} \wedge h+g_{n} \vee h\right) / 2 \leqslant g$ for all $n$ and so $f \leqslant k \leqslant g$. If $f(x)<g(x)$, then for some $n, f(x)<\left(f_{n} \wedge h+g_{n} \vee h\right)(x) / 2$ $<g(x)$ and so $f(x)<k(x)<g(x)$.

(only if). Clearly, $K$ interposes $(L, M)$. Let $A \prec_{L} B$. Let $f \in L$ be such that $1_{A} \leqslant f \leqslant 1_{B}$. Since $f \in L$ and $f \leqslant 1 \in M$, there is an $h \in K$ such that $f \leqslant$ $h \leqslant 1$ and $f(x)<h(x)<1$ whenever $f(x)<1 . A \subset N(h-1) \subset B$ and so $A$ $\prec_{N(K)} B$. Now let $A \prec_{M} B$. Let $g \in M$ be such that $1_{A} \leqslant g \leqslant 1_{B}$. Since $g \in M$ and $g \geqslant 0 \in L$, there is a $k \in K$ such that $0 \leqslant k \leqslant g$ and $0<k(x)<g(x)$ whenever $0<g(x) . A \subset P(k) \subset B$ and so $A \prec_{p(K)} B$.

In the special case that $K=L \cap M$, we can replace (2) by a weaker condition.

3.16. THEOREM. Let $X$ be $a$ set and let $L$ and $M$ be closed lattice cones on $X$. Then $L \cap M$ ultrastrictly interposes $(L, M)$ iff

(1) $L \cap M$ interposes $(L, M)$,

(2) $\prec_{L} \subset \prec_{N(M)}$ and $\prec_{M} \subset \prec_{P(L)}$.

Proof. Since $N(L \cap M) \subset N(M)$ and $P(L \cap M) \subset P(L)$, condition (2) of 3.15 implies (2) of 3.16. Now suppose (1) and (2) hold. Let $A \prec_{L} B$. Let $f \in$ $L$ be such that $1_{A} \leqslant f \leqslant 1_{B} . \quad L \subset \overline{\mathrm{cn}}(N(M))$ and so there is a sequence $\left\{g_{n}\right\}_{n \in \mathrm{N}}$ in $M$ which decreases to $f$. By (1) there is an $h_{n} \in L \cap M$ such that $f \leqslant h_{n} \leqslant g_{n}$. 
$\left\{\bigwedge_{k=1}^{n} h_{k}\right\}_{n \in \mathrm{N}}$ decreases to $f$ and so $f \in \overline{\mathrm{cn}}(N(L \cap M))$. Thus $A \prec_{N(L \cap M)} B$. Likewise one sees that if $A \prec_{M} B$, then $A \prec_{p(L \cap M)} B$.

3.17. Corollary. Let $X$ be $a$ set and let $A$ and $B$ be sublattices of $2^{X}$ to which $\varnothing$ and $X$ belong. Then $\overline{\mathrm{cn}}(A) \cap \overline{\mathrm{cn}}(B)$ ultrastrictly interposes $(\overline{\mathrm{cn}}(A)$, $\overline{\mathrm{cn}}(B))$ iff

(1) $\overline{\mathrm{cn}}(A) \cap \overline{\mathrm{cn}}(B)$ interposes $(\overline{\mathrm{cn}}(A), \overline{\mathrm{cn}}(B))$,

(2) $A \subset B_{\delta}$ (= intersections of sequences in $\left.B\right)$ and $B \subset A_{\sigma}$ (= unions of sequences in $A)$.

Proof. Suppose (1) and (2) hold. Set $L=\overline{\operatorname{cn}}(A)$ and $M=\overline{\operatorname{cn}}(B)$. We must show that $A \subset N(M)$ and $B \subset P(L)$. Let $A \in A$. By (2) there is a sequence $\left\{B_{n}\right\}_{n \in \mathrm{N}}$ in $B$ such that $A=\bigcap_{n \in \mathrm{N}} B_{n}$. Let $f_{n} \in \overline{\mathrm{cn}}(A) \cap \overline{\mathrm{cn}}(B)$ be such that $1_{A} \leqslant f_{n} \leqslant 1_{B_{n}}$. Then $A=\bigcap_{n \in N} N\left(f_{n}-1\right) \in N(M)$. Likewise it is seen that $B \subset P(L)$. Now suppose (1) and (2) of 3.16 hold. We show that $N(M) \subset B_{\delta}$. Let $f \in M$. For each $n \in \mathbf{N}$, there is a $B_{n} \in B$ such that $N(f)=\{x: f(x) \geqslant 0\}$ $\subset B_{n} \subset\{x: f(x) \geqslant-1 / n\}$. Clearly, $N(f)=\bigcap_{n \in \mathbb{N}} B_{n} \in B_{\delta}$. Likewise one sees that $P(L) \subset A_{\sigma}$.

The 'if' half of 3.17 was proved in [2] and the 'only if' half was to have been announced there.

4. Quasi-proximity spaces and the Császár Compactification Theorem. In this section we derive a number of properties of quasi-proximity spaces-most of which are necessary for the proof of the Császár theorem, but some of which are of interest in themselves-and, of course, we prove the Császár Compactification Theorem. Our basic theme is that just about every property of a quasi-proximity space $(X, \delta)$ can be expressed in terms of the lattice cone $C(X, \delta)$ and, as a rule, quite neatly expressed. A case in point is Proposition 4.7.

We begin with a formalization of this basic theme in the language of categories. The category of quasi-proximity spaces has for its objects, naturally, quasi-proximity spaces, and for $(X, \delta)$ and $(Y, \eta)$ quasi-proximity spaces, the morphisms of $(X, \delta)$ to $(Y, \eta)$ are those mappings $\varphi: X \rightarrow Y$ which are such that for $A, B \subset X$, if $A \delta B$, then $\varphi[A] \eta \varphi[B]$. We call such mappings quasi-proximity mappings. We can also form a category $L$ whose objects are pairs $(X, K), X$ a set and $K$ a closed lattice cone on $X$, and for $(X, K)$ and $(Y, L)$ objects in $L$, the morphisms of $(X, K)$ to $(Y, L)$ are those mappings $\varphi: X \rightarrow Y$ such that $f \circ \varphi$ $\in K$ for all $f \in L$. We shall see that the category $L$ and the category of quasiproximity spaces are isomorphic. However, before pursuing this further, it will be convenient to have reformulations, in terms of quasi-proximities, of the definition of $C(X, \delta)$ and of (i) of the Characterization Theorem. 
4.1. Proposition. Let $(X, \delta)$ be a quasi-proximity space.

(i) For $f \in B(X), f \in C(X, \delta)$ iff inf $f[A] \leqslant \sup f[B]$ whenever $A \delta B$.

(ii) For $A, B \subset X, A \delta B$ iff $\inf f[A] \leqslant \sup f[B]$ whenever $f \in C(X, \delta)$.

Proof. We first prove that if $f \in C(X, \delta)$ and $A \delta B$, then inf $f[A] \leqslant$ sup $f[B]$. Recall that $C(X, \delta)=C(X, \prec)$, where $\prec$ is the idempotent inclusion on $X$ obtained from $\delta$ via (IQ) (in $\S 1$ ). Suppose inf $f[A]=s>r>\sup f[B]$. $A \subset\{x: f(x) \geqslant s\}<\{x: f(x) \geqslant r\} \subset X \sim B$ so that $A<X \sim B$, i.e. $A \bar{\delta} B$. This takes care of the 'only if' parts of (i) and (ii). Now let $f \in B(X)$ be such that $\inf f[A] \leqslant \sup f[B]$ whenever $A \delta B$. Let $r, s \in \mathbf{R}, r<s$. Set $A=\{x: f(x)$ $\geqslant s\}$ and $B=\{x: f(x) \geqslant r\}$. Since inf $f[A] \geqslant s>r \geqslant \sup f[X \sim B], A \bar{\delta}(X \sim B)$, i.e. $A \prec B$. Thus (i) is proved. Now let $A \bar{\delta} B$. $A \prec X \sim B$ and so by the Characterization Theorem there is an $f \in C(X, \delta)$ such that $1_{A} \leqslant f \leqslant 1_{X \sim B}$. inf $f[A]$ $=1>0=\sup f[B]$.

4.2. Proposition. Let $(X, \delta)$ and $(Y, \eta)$ be quasi-proximity spaces, and let $\varphi: X \rightarrow Y$. Then the following conditions are equivalent.

(a) $\varphi$ is a quasi-proximity mapping.

(b) $f \in C(Y, \eta) \Rightarrow f \circ \varphi \in C(X, \delta)$.

Proof. (a) $\Rightarrow(\mathrm{b})$. Let $f \in C(Y, \eta)$. Let $A \delta B . \varphi[A] \eta \varphi[B]$ so that by 4.1(i) inf $f[\varphi[A]] \leqslant \sup f[\varphi[B]] . f[\varphi[A]]=(f \circ \varphi)[A]$ and $f[\varphi[B]]=$ $(f \circ \varphi)[B]$ and so $\inf (f \circ \varphi)[A] \leqslant \sup (f \circ \varphi)[B]$. Again by 4.1(i) we have $f \circ \varphi$ $\in C(X, \delta)$.

(b) $\Rightarrow$ (a). Let $A \delta B$. For $f \in C(Y, \eta)$, inf $f[\varphi[A]]=\inf (f \circ \varphi)[A] \leqslant$ $\sup (f \circ \varphi)[B]=\sup f[\varphi[B]]$ and so by $4.1(\mathrm{ii}) \varphi[A] \eta \varphi[B]$.

The isomorphism of the category of quasi-proximity spaces and the category $L$ is now easily constructed via 4.2 and the Characterization Theorem. It is also easy to see that this isomorphism induces an isomorphism between the full subcategories whose objects are proximity spaces (i.e. quasi-proximity spaces whose quasi-proximities are symmetric) and those objects $(X, K)$ of $L$ for which $K$ is a closed vector sublattice of $B(X)$ which contains the constant functions (see [5]).

There are three natural topologies on a quasi-proximity space, and these we now discuss. Let $(X, \delta)$ be a quasi-proximity space. For $A \subset X, \operatorname{cl}_{\delta}(A)$ is defined to be $\{x \in X:\{x\} \delta A\}$. The relation $\delta^{-1}(=\{(A, B): B \delta A\})$ is also a quasi-proximity on $X$ and so defines an operator $\mathrm{cl}_{\delta}-1$. We wish to prove that $\mathrm{cl}_{\delta}$ and $\mathrm{cl}_{\delta-1}$ are Kuratowski closure operators on $X$, but first we need a lemma.

4.3. Lemma. Let $(X, \delta)$ be a quasi-proximity space, and let $A, B \subset X$. If $A \delta \mathrm{cl}_{\delta}(B)$, then $A \delta B$. 
Proof. Let $f \in C(X, \delta)$. If $x \in \mathrm{cl}_{\delta}(B)$, then $f(x) \leqslant \sup f[B]$. Thus $\sup f\left[\mathrm{cl}_{\delta}(B)\right] \leqslant \sup f[B]$. Since $A \delta \operatorname{cl}_{\delta}(B)$ we have $\inf f[A] \leqslant \sup f\left[\mathrm{cl}_{\delta}(B)\right]$ and so $\inf f[A] \leqslant \sup f[B]$. By 4.1(ii) $A \delta B$.

4.4. Corollary. $A \delta B$ if $\mathrm{cl}_{\delta-1}(A) \delta \mathrm{cl}_{\delta}(B)$. $\Rightarrow A \delta B$.

Proof. $\mathrm{cl}_{\delta-1}(A) \delta \mathrm{cl}_{\delta}(B) \Rightarrow \mathrm{cl}_{\delta}-1(A) \delta B \Rightarrow B \delta^{-1} \mathrm{cl}_{\delta}-1(A) \Rightarrow B \delta^{-1} A$

4.5. Proposition. $\left({ }^{6}\right)$ Let $(X, \delta)$ be a quasi-proximity space. Then $\mathrm{cl}_{\delta}$ and $\mathrm{cl}_{\delta}-1$ are Kuratowski closure operators on $X$.

PROOF. We must show

(1) $\mathrm{cl}_{\delta}(\varnothing)=\varnothing$,

(2) $A \subset \mathrm{cl}_{\delta}(A)$,

(3) $\mathrm{cl}_{\delta}(A \cup B)=\mathrm{cl}_{\delta}(A) \cup \mathrm{cl}_{\delta}(B)$,

(4) $\operatorname{cl}_{\delta}(A)=\mathrm{cl}_{\delta}\left(\mathrm{cl}_{\delta}(A)\right)$.

(1) follows from $\left(\mathrm{Q}_{1}\right),(2)$ from $\left(\mathrm{Q}_{2}\right)$ and (3) from $\left(\mathrm{Q}_{4}\right)$. By (2) $\mathrm{cl}_{\delta}(A) \subset$ $\mathrm{cl}_{\delta}\left(\mathrm{cl}_{\delta}(A)\right)$. Let $x \in \mathrm{cl}_{\delta}\left(\mathrm{cl}_{\delta}(A)\right)$. $\{x\} \delta \mathrm{cl}_{\delta}(A)$ and so by $4.3\{x\} \delta A$, i.e. $x \in$ $\mathrm{cl}_{\delta}(A)$. Since $\delta^{-1}$ is also a quasi-proximity we are done.

4.6. Definition. Let $(X, \delta)$ be a quasi-proximity space. The topologies defined by $\mathrm{cl}_{\delta}$ and $\mathrm{cl}_{\delta-1}$ are denoted, respectively, by $\tau_{l}(\delta)$ and $\tau_{r}(\delta)$. The supremum of $\tau_{l}(\delta)$ and $\tau_{r}(\delta)$ is denoted by $\tau(\delta)$ and is called the topology of $(X, \delta)$.

4.7. Proposition. Let $(X, \delta)$ be a quasi-proximity space.

(i) The sets of the form $\{x: f(x)>0\}, f \in C(X, \delta)$, form a base for $\tau_{l}(\delta)$

(ii) The sets of the form $\{x: f(x)<0\}, f \in C(X, \delta)$, form a base for $\tau_{r}(\delta)$

(iii) The sets of the form $\{x: f(x)>0>g(x)\}, f, g \in C(X, \delta)$, form a base for $\tau(\delta)$.

Consequently, $\tau(\delta)$ (resp. $\tau_{l}(\delta)$, resp. $\left.\tau_{r}(\delta)\right)$ is the smallest topology on $X$ which renders the members of $C(X, \delta)$ continuous (resp. lower semicontinuous, resp. upper semicontinuous).

Proof. (i) Let $x \in U \in \tau_{l}(\delta) . x \notin \mathrm{cl}_{\delta}(X \sim U)$ and so $\{x\} \bar{\delta}(X \sim U)$. By 4.1(ii) there is a $g \in C(X, \delta)$ such that $g(x)>s=\sup g[X \sim U]$. Set $f=$ $g$-s. Then $x \in\{y: f(y)>0\} \subset U$. Clearly, $\{y: f(y)>0\} \in \tau_{l}(\delta)$.

(ii) follows from (i) and the observations that $\tau_{r}(\delta)=\tau_{l}\left(\delta^{-1}\right)$ and $C\left(X, \delta^{-1}\right)$ $=-C(X, \delta)$.

(6) See footnote 4 . 
(iii) follows from (i), (ii) and the observation that the sets of the form $U \cap$ $V, U \in \tau_{l}(\delta), V \in \tau_{r}(\delta)$ form a base for $\tau(\delta)$.

A natural conjecture is that $C(X, \delta)$ consists of those $f \in B(X)$ such that $f$ is $\tau_{l}(\delta)$-lower semicontinuous and $\tau_{r}(\delta)$-upper semicontinuous. Natural, but, lamentably, false.

4.8. Proposition. Let $(X, \delta)$ and $(Y, \eta)$ be quasi-proximity spaces, and let $\varphi: X \rightarrow Y$ be a quasi-proximity mapping. Then $\varphi$ is continuous.

Proof. Let $x \in X$ and let $U$ be a neighborhood of $\varphi(x)$. By 4.7(iii) there are $f, g \in C(Y, \eta)$ such that $\varphi(x) \in\{y \in Y: f(y)>0>g(y)\} \subset U$. $x \in V=$ $\{w \in X:(f \circ \varphi)(w)>0>(g \circ \varphi)(w)\}, \varphi[V] \subset U$, and by 4.2 and 4.7(iii), $V \in$ $\tau(\delta)$.

The following lemma serves as motivation as well as preparation for the proof of the Császár Compactification Theorem.

4.9. Lemma. Let $(X, \delta)$ be a compact Hausdorff quasi-proximity space and let $y: C(X, \delta) \rightarrow \mathrm{R}$ satisfy

(1) $y$ is additive and $\mathbf{R}^{+}$-homogeneous,

(2) $y$ preserves the lattice operations,

(3) $y$ preserves constants, i.e. for $c \in \mathbf{R}, y\left(\mathrm{cl}_{X}\right)=c$.

Then there is a unique $x \in X$ such that $y(f)=f(x)$ for all $f \in C(X, \delta)$.

Proof. For $f \in C(X, \delta)$, set $A_{f}=\{x \in X: y(f)=f(x)\}$. The $A_{f}$ are clearly closed and we assert that $\left\{A_{f}: f \in C(X, \delta)\right\}$ has the finite intersection property. Let $f_{1}, \ldots, f_{n} \in C(X, \delta)$ and suppose that $\bigcap_{i=1}^{n} A_{f_{i}}=\varnothing$. For any $x \in X$, there is an $i$ such that $y\left(f_{i}\right) \neq f_{i}(x)$, i.e. $\max \left\{f_{i}(x), y\left(f_{i}\right)\right\}>$ $\min \left\{f_{i}(x), y\left(f_{i}\right)\right\}$. Set $f=\Sigma_{i=1}^{n} f_{i} \vee y\left(f_{i}\right)$ and $g=\Sigma_{i=1}^{n} f_{i} \wedge y\left(f_{i}\right)$. For each $x \in X, f(x)>g(x)$ and so there is a $c>0$ such that $g+c \leqslant f . y(f)=\sum_{i=1}^{n} y\left(f_{i}\right)$ $=y(g)$, and $y(g)+c=y(g+c) \leqslant y(f)$, a contradiction. Thus there is an $x \in$ $\bigcap\left\{A_{f}: f \in C(X, \delta)\right\}$. Obviously, $f(x)=y(f)$ for all $f \in C(X, \delta)$. Since $C(X, \delta)$ separates points there is at most one such $x$.

4.10. CsÁszár Compactification Theorem. Let $(X, \delta)$ be a quasi-proximity space.

(i) $(X, \delta)$ has a compactification.

(ii) If $(Y, \eta)$ is a compact Hausdorff quasi-proximity space, and if $\kappa: X$

$\rightarrow Y$ is a quasi-proximity mapping, then the following conditions are equivalent.

(a) $((Y, \eta), \kappa)$ is a compactification of $(X, \delta)$.

(b) For every $f \in C(X, \delta)$, there is a unique $g \in C(Y, \eta)$ such that $f=g \circ \kappa$.

(c) If $(Z, \zeta)$ is a compact Hausdorff quasi-proximity space, and if 
$\varphi: X \rightarrow Z$ is a quasi-proximity mapping, then there is a unique quasi-proximity mapping $\psi: Y \rightarrow Z$ such that $\varphi=\psi \circ \kappa$.

(iii) If $\left(\left(Y_{1}, \eta_{1}\right), \kappa_{1}\right)$ and $\left(\left(Y_{2}, \eta_{2}\right), \kappa_{2}\right)$ are compactifications of $(X, \delta)$, then there is a bijection $\varphi: Y_{1} \rightarrow Y_{2}$ such that $\kappa_{2}=\varphi \circ \kappa_{1}$ and $\varphi$ and $\varphi^{-1}$ are quasi-proximity mappings.

Proof. (i) Let $Y$ consist of all $y: C(X, \delta) \rightarrow \mathrm{R}$ which satisfy (1)-(3) of 4.9. Define $\kappa: X \rightarrow Y$ by

$$
\kappa(x)(f)=f(x), \quad f \in C(X, \delta), x \in X .
$$

For $f \in C(X, \delta)$, define $\hat{f} \in B(Y)$ by

$$
\hat{f}(y)=y(f), \quad y \in Y,
$$

and observe that $\hat{f} \circ k=f$. The mapping $f \mapsto \hat{f}$ is easily seen to be additive and $\mathbf{R}^{+}$-homogeneous and to preserve the lattice operations and the constant functions. We claim that it is also distance-preserving. Let $f, g \in C(X, \delta)$.

$$
\|\hat{f}-\hat{g}\| \geqslant \sup \{|(\hat{f}-\hat{g})(k(x))|: x \in X\}=\sup \{|(f-g)(x)|: x \in X\}=\|f-g\| .
$$

Set $c=\|f-g\|$. $f-c \leqslant g \leqslant f+c$ and so $\hat{f}-c \leqslant \hat{g} \leqslant \hat{f}+c$, i.e. $\|\hat{f}-\hat{g}\| \leqslant c$. Thus the set $K=\{\hat{f}: f \in C(X, \delta)\}$ is a closed lattice cone on $Y$ and so defines a quasi-proximity $\eta$ on $Y$. We now proceed to show that $((Y, \eta), \kappa)$ is a compactification of $(X, \delta)$. $K$ obviously separates the points of $Y$ and so $\tau(\eta)$ is Hausdorff. Let $\left\{y_{i}\right\}_{i \in I}$ be a net in $Y$. It is a simple consequence of the Tychonoff product theorem that $\left\{y_{i}\right\}_{i \in I}$ has a subnet $\left\{y_{i_{j}}\right\}_{j \in J}$ such that $\left\{y_{i_{j}}(f)\right\}_{j \in J}$ converges for all $f \in C(X, \delta)$. The function $y: f \mapsto \lim _{j} y_{i_{j}}(f)$ belongs to $Y$ and by $4.7,\left\{y_{i_{j}}\right\}_{j \in J} \tau(\eta)$-converges to $y$. Thus $(Y, \eta)$ is compact Hausdorff. Let $A, B \subset X$. By 4.1(ii) and the identity $\hat{f} \circ \kappa=f$ we have:

$$
\begin{aligned}
A \delta B & \Longleftrightarrow \inf f[A] \leqslant \sup f[B] \quad \text { all } f \in C(X, \delta) \\
& \Longleftrightarrow \inf \hat{f}[\kappa[A]] \leqslant \sup \hat{f}[\kappa[B]] \quad \text { all } f \in C(X, \delta) \\
& \Longleftrightarrow \inf k[\kappa[A]] \leqslant \sup k[\kappa[B]] \quad \text { all } k \in K \\
& \Longleftrightarrow \kappa[A] \eta \kappa[B] .
\end{aligned}
$$

Thus $\kappa[A] \eta \kappa[B]$ iff $A \delta B$. It remains only to prove that $\kappa[X]$ is dense in $Y$. Let $U$ be a nonvoid open subset of $Y$ and suppose $U \cap \kappa[X]=\varnothing$. By 4.7 there are $f, g \in C(X, \delta)$ such that $\varnothing \neq\{y \in Y: \hat{f}(y)>0>\hat{g}(y)\} \subset U$. Since $U \cap$ $\kappa[X]=\varnothing$, for each $x \in X$, either $\hat{f}(\kappa(x)) \leqslant 0$ or $\hat{g}(\kappa(x)) \geqslant 0$. It follows that $(-f) \vee g \geqslant 0$. Therefore $f \leqslant f+(-f) \vee g=0 \vee(f+g)$ and so $\hat{f} \leqslant 0 \vee$ $(\hat{f}+\hat{g})$. There is a $y \in Y$ such that $\hat{f}(y)>0>\hat{g}(y) . \quad 0<\hat{f}(y) \leqslant$ $\max (0, \hat{f}(y)+\hat{g}(y))$ implies that $\hat{f}(y)+\hat{g}(y) \geqslant \hat{f}(y)$ which in turn implies that 
$\ddot{g}(y) \geqslant 0$, a contradiction Thus $((Y, \eta), \kappa)$ is a compactification of $(X, \delta)$.

(ii) Let $(Y, \eta)$ be a compact Hausdorff quasi-proximity space, and let $\kappa$ : $X \rightarrow Y$ be a quasi-proximity mapping.

(a) $\Rightarrow\left(\right.$ b). The mapping $f \mapsto f \circ K$ is additive and $\mathbf{R}^{+}$-homogeneous, preserves the lattice operations and constant functions, and, since $\kappa[X]$ is dense in $Y$, preserves distance. Thus the set $K=\{f \circ k: f \in C(Y, \eta)\}$ is a closed lattice cone on $X$ and so defines a quasi-proximity $\delta^{\prime}$ on $X$. Since $K=C\left(X, \delta^{\prime}\right)$ we shall be done once we show that $\delta=\delta^{\prime}$. Let $A, B \subset X$.

$A \delta B \Longleftrightarrow \kappa[A] \eta \kappa[B] \Longleftrightarrow \inf f[\kappa[A]] \leqslant \sup f[\kappa[B]] \quad$ for all $f \in C(Y, \eta)$ $\Longleftrightarrow \inf k[A] \leqslant \sup k[B] \quad$ for all $k \in K \Longleftrightarrow A \delta^{\prime} B$.

(b) $\Rightarrow$ (c). For $f \in C(X, \delta)$, let $\hat{f}$ be the member of $C(Y, \eta)$ such that $f=$ $\hat{f} \circ \kappa$. Let $(Z, \zeta)$ be a compact Hausdorff quasi-proximity space, and let $\varphi: X \rightarrow$ $Z$ be a quasi-proximity mapping. For $y \in Y$, the mapping $f \mapsto(f \circ \varphi)^{\wedge}(y)$ from $C(Z, \zeta)$ to $\mathbf{R}$ is additive and $\mathbf{R}^{+}$-homogeneous and preserves the lattice operations and constant functions. By 4.9 there is a unique $\psi(y) \in Z$ such that $(f \circ \varphi)^{\wedge}(y)$ $=f(\psi(y))$ for all $f \in C(Z, \zeta)$. For $f \in C(Z, \zeta),(f \circ \varphi)^{\wedge} \in C(Y, \eta)$ and so by $4.2, \psi$ is a quasi-proximity mapping. Let $x \in X$. For $f \in C(Z, \zeta)$,

$$
f((\psi \circ \kappa)(x))=(f \circ \varphi)^{\wedge}(\kappa(x))=(f \circ \varphi)(x)=f(\varphi(x)) .
$$

Since $C(Z, \zeta)$ separates the points of $Z$, we have $(\psi \circ \kappa)(x)=\varphi(x)$. Thus $\psi \circ \kappa$ $=\varphi$. Suppose $\theta: Y \rightarrow Z$ is a quasi-proximity mapping such that $\theta \circ \kappa=\varphi$. For $f \in C(Z, \zeta), f \circ \theta \in C(Y, \eta)$ and $(f \circ \theta) \circ \kappa=f \circ \varphi$ so that $f \circ \theta$ must be $(f \circ \varphi)^{n}$. It follows that $f(\theta(y))=f(\psi(y))$ for all $f \in C(Z, \zeta)$ and $y \in Y$, i.e. $\theta=\psi$.

(c) $\Rightarrow$ (a). By (i) $(X, \delta)$ has a compactification, say $\left(\left(Y^{\prime}, \eta^{\prime}\right), \kappa^{\prime}\right)$. We know that (a) $\Rightarrow$ (c) and so there is a unique quasi-proximity mapping $\psi^{\prime}: Y^{\prime} \rightarrow Y$ such that $\psi^{\prime} \circ \kappa^{\prime}=\kappa$. By hypothesis there is a unique $\psi: Y \rightarrow Y^{\prime}$ such that $\kappa^{\prime}=\psi \circ \kappa . \psi^{\prime} \circ \psi$ and id $_{Y}$ (= the identity mapping of $Y$ to $Y$ ) are quasi-proximity mappings of $Y$ to itself such that $\left(\psi^{\prime} \circ \psi\right) \circ \kappa=\mathrm{id}_{Y} \circ \kappa=\kappa$ and so $\psi^{\prime} \circ \psi$ $=\mathrm{id}_{Y}$. Likewise $\psi \circ \psi^{\prime}=\mathrm{id}_{Y^{\prime}}$. It follows that $((Y, \eta), \kappa)$ is a compactification of $(X, \delta)$.

(iii) is a consequence of the implication '(a) $\Rightarrow$ (c)' in (ii).

4.11. Corollary. Let $(X, \delta)$ be a quasi-proximity space, and let $((Y, \eta), \kappa)$ be a compactification of $(X, \delta)$.

(i) $k$ is a continuous open mapping of $X$ onto $k[X]$.

(ii) $\kappa$ is injective iff $(X, \delta)$ is Hausdorff.

Proof. (i) $\kappa$ is continuous by 4.8. Let $x \in U \in \tau(\delta)$. We must show the existence of a $V \in \tau(\eta)$ such that $\kappa(x) \in V \cap \kappa[X] \subset \kappa[U]$. By 4.7(iii) there 
exist $f, g \in C(X, \delta)$ such that $x \in\{w \in X: f(w)>0>g(w)\} \subset U$. By (ii) of 4.10 there exist $\hat{f}, \hat{g} \in C(Y, \eta)$ such that $f=\hat{f} \circ \kappa$ and $g=\hat{g} \circ \kappa$. Set $V=$ $\{y \in Y: \hat{f}(y)>0>\hat{g}(y)\}$.

(ii) For $x, y \in X, \kappa(x)=\kappa(y) \Longleftrightarrow\{\kappa(x)\} \eta\{\kappa(g)\}$ and $\{\kappa(y)\} \eta\{\kappa(x)\}$ $\Longleftrightarrow\{x\} \delta\{y\}$ and $\{y\} \delta\{x\} \Longleftrightarrow f(x)=f(y)$ for all $f \in C(X, \delta)$. Since $(X, \delta)$ is Hausdorff iff $C(X, \delta)$ separates points, we are done.

5. Ordered topological spaces and their compactification. The results in this section were announced in [4]. We begin with some definitions and elementary facts about ordered topological spaces.

5.1. Definition. An ordered topological space is a triple $(X, \tau, \preccurlyeq)$ consisting of a set $X$, a topology $\tau$ on $X$, and an order $\leqslant$ on $X$, that is, a transitive, reflexive, antisymmetric binary relation $\leqslant$ on $X$, which is closed, that is, $\leqslant$ is a closed subset of $X \times X$ when the latter is given the product topology.

5.2. Proposition. The topology of an ordered topological space is Hausdorff.

Proof. $[15$, p. 25].

5.3. Definition. Let $X$ be an ordered topological space (whenever possible we shall omit explicit mention of the topology of an ordered topological space and shall denote the order by $\preccurlyeq$. An order compactification of $X$ is a pair $(Y, k)$ consisting of a compact ordered topological space $Y$ and a mapping $\kappa: X \rightarrow Y$ such that

- $k$ is a topological embedding,

- $\kappa$ is an order embedding, i.e. $\kappa(x) \leqslant \kappa(y)$ iff $x \leqslant y$,

- $k[X]$ is dense in $Y$.

We shall call two order compactifications $\left(Y_{1}, \kappa_{1}\right)$ and $\left(Y_{2}, \kappa_{2}\right)$ of $X$ equivalent if there is a mapping $\varphi: Y_{1} \rightarrow Y_{2}$ such that $\kappa_{2}=\varphi \circ \kappa_{1}$ and $\varphi$ is an order homeomorphism, that is, $\varphi$ is an order embedding and a homeomorphism.

It is our object to determine which ordered topological spaces have order compactifications and to descrile the order compactifications of such spaces. The key to the construction and classification of order compactifications is the notion of a compatible quasi-proximity, which we now proceed to define. We first show that if $(X, \delta)$ is a Hausdorff quasi-proximity space, there is a natural order $\leqslant_{\delta}$ on $X$ such that $\left(X, \tau(\delta), \leqslant_{\delta}\right)$ is an ordered topological space.

5.4. Proposition. Let $(X, \delta)$ be a quasi-proximity space. Then the binary relation $\leqslant_{\delta}$ on $X$ defined by

$$
x \leqslant \delta \Leftrightarrow\{x\} \delta\{y\}
$$


is transitive, reflexive, closed with respect to $\tau(\delta)$, and, if $(X, \delta)$ is Hausdorff, is antisymmetric.

Proof. By 4.1(ii) $x \leqslant \delta$ iff $f(x) \leqslant f(y)$ for all $f \in C(X, \delta)$. It follows immediately that $\leqslant_{\delta}$ is transitive and reflexive and, since the members of $C(X, \delta)$ are $\tau(\delta)$-continuous, closed. Since $(X, \delta)$ is Hausdorff iff $C(X, \delta)$ separates points, $\leqslant_{\delta}$ is antisymmetric if $(X, \delta)$ is Hausdorff.

5.5. Definition. Let $X$ be an ordered topological space. We shall call a quasi-proximity $\delta$ on $X$ compatible if $\tau(\delta)$ is the topology of $X$ and $\leqslant_{\delta}$ is the order of $X$.

If $X$ is an ordered topological space, $\delta$ a compatible quasi-proximity on $X$, and if $((Y, \eta), \kappa)$ is a compactification of $(X, \delta)$, then $\left(\left(Y, \tau(\eta), \leqslant_{\eta}\right), \kappa\right)$ is an order compactification of $X: \kappa$ is a topological embedding by 4.11 , and obviously $\boldsymbol{K}$ is an order embedding. Thus, if an ordered topological space has a compatible quasi-proximity, then it has an order compactification. The converse of this statement is also true, but before taking up this matter we prove the crucial result that a compact ordered topological space has exactly one compatible quasiproximity. We begin with the simpler problem of unicity.

5.6. Definition. Let $X$ be an ordered topological space. A function $f: X$ $\rightarrow \mathbf{R}$ is increasing if $f(x) \leqslant f(y)$ whenever $x \leqslant y . \quad C(X, \leqslant)$ denotes the set of all bounded, continuous increasing functions on $X$.

5.7. Proposition. Let $X$ be an ordered topological space.

(i) $C(X, \leqslant)$ is a closed lattice cone on $X$.

(ii) If $\delta$ is a compatible quasi-proximity on $X$, then $C(X, \delta) \subset C(X, \leqslant)$.

(iii) If $X$ has a compatible quasi-proximity, then the quasi-proximity defined by $C(X, \preccurlyeq$ is also compatible.

PRoof. (i) is easily verified.

(ii) The members of $C(X, \delta)$ are $\leqslant_{\delta}$-increasing, and by 4.7(iii) they are $\tau(\delta)$-continuous. By the definition of compatibility $C(X, \delta) \subset C(X, \preccurlyeq)$.

(iii) Let $\delta$ be a compatible quasi-proximity on $X$, and let $\eta$ be the quasiproximity defined by $C(X, \Im)$. By the Characterization Theorem $C(X, \eta)=$ $C(X, \leqslant)$. By 4.7(iii) $\tau(\eta)$ is the smallest topology on $X$ which renders the members of $C(X, \preccurlyeq$ continuous. $\tau(\delta)$ is the topology of $X$ and so $\tau(\delta) \supset \tau(\eta)$. On the other hand $C(X, \delta) \subset C(X, \lessgtr)$ and so the members of $C(X, \delta)$ are $\tau(\eta)$-continuous. Thus, again by 4.7(iii), $\tau(\eta) \supset \tau(\delta)$. Thus $\tau(\eta)$ is the topology of $X$. If $x \leqslant \eta$, then $f(x) \leqslant f(y)$ for all $f \in C(X, \leqslant$ and in particular for all $f \in C(X, \delta)$, i.e. $x \leqslant_{\delta} y$. Thus $\leqslant_{\eta}$ is the order of $X$.

It follows from (iii) of 5.7 that if $X$ is an ordered topological space which has a unique compatible quasi-proximity, then that compatible quasi-proximity 
must be the one defined by $C(X, \leqslant)$. That a compact ordered topological space has at most one compatible quasi-proximity will follow from the Kakutani-Stone Theorem (5.9 below). We shall deduce the Kakutani-Stone Theorem from the following result which will also be useful in the next section.

5.8. Translation Lemma. Let $(X, \tau)$ be a compact topological space, and let $\delta$ be a quasi-proximity on $X$ such that $\tau(\delta) \subset \tau$. Then for $A, B \subset X$, $A \delta B$ iff there exist $x \in \operatorname{cl}_{\tau}(A)$ and $y \in \operatorname{cl}_{\tau}(B)$ such that $\{x\} \delta\{y\}$.

Proof. If we are given $x \in \operatorname{cl}_{\tau}(A)$ and $y \in \operatorname{cl}_{\tau}(B)$ such that $\{x\} \delta\{y\}$, then $\{x\} \subset \mathrm{cl}_{\tau}(A) \subset \mathrm{cl}_{\delta-1}(A)$, and $\{y\} \subset \mathrm{cl}_{\tau}(B) \subset \mathrm{cl}_{\delta}(B)$ so that $\mathrm{cl}_{\delta-1}(A) \delta$ $\mathrm{cl}_{\delta}(B)$. By $4.4 A \delta B$.

Now suppose $\{x\} \bar{\delta}\{y\}$ for all $x \in \mathrm{cl}_{\tau}(A), y \in \mathrm{cl}_{\tau}(B)$. Set $A^{-}=\mathrm{cl}_{\tau}(A)$, $B^{-}=\mathrm{cl}_{\tau}(B)$. Let $x \in A^{-}$. For $y \in B^{-}$, there is, by $\left(Q_{5}\right)$, a $C_{y} \subset X$ such that $\{x\} \bar{\delta} C_{y}$ and $\left(X \sim C_{y}\right) \bar{\delta}\{y\} . y \in X \sim \mathrm{cl}_{\delta-1}\left(X \sim C_{y}\right)=U_{y} \in \tau_{r}(\delta) \subset \tau$. Thus $\left\{U_{y}: y \in B^{-}\right\}$is a $\tau$-open cover of $B^{-}$and so there exist $y_{1}, \ldots, y_{n} \in B^{-}$ such that $B^{-} \subset \bigcup_{i=1}^{n} U_{y_{i}} \cdot U_{y_{i}} \subset C_{y_{i}}$ and so $\{x\} \bar{\delta} U_{y_{i}}, i=1, \ldots, n$. By $\left(Q_{4}\right)\{x\} \bar{\delta} \bigcup_{i=1}^{n} U_{y_{i}}$, and hence $\{x\} \bar{\delta} B$. Thus for each $x \in A^{-}$, there is a $D_{x}$ $\subset X$ such that $\{x\} \bar{\delta} D_{x}$ and $\left(X \sim D_{x}\right) \bar{\delta} B . x \in X \sim \mathrm{cl}_{\delta}\left(D_{x}\right)=V_{x} \in \tau_{l}(\delta) \subset \tau$. Thus $\left\{V_{x}: x \in A^{-}\right\}$is a $\tau$-open cover of $A^{-}$and so there exist $x_{1}, \ldots, x_{m} \in$ $A^{-}$such that $A^{-} \subset \bigcup_{i=1}^{m} V_{x_{i}} . V_{x_{i}} \subset X \sim D_{x_{i}}$, and so $V_{x_{i}} \bar{\delta} B, i=1, \ldots, m$. By $\left(\mathrm{Q}_{3}\right)\left(\bigcup_{i=1}^{m} V_{x_{i}}\right) \bar{\delta} B$ and hence $A \bar{\delta} B$.

5.9. KaKutAni-Stone TheOREM. Let $X$ be a compact topological space, and let $K$ be a lattice cone of continuous functions on $X$. Then $\mathrm{cl}(K)$ consists of all $f \in C(X)$ such that $f(x) \leqslant f(y)$ whenever $x, y \in X$ and $g(x) \leqslant g(y)$ for all $g$ $\in K$.

Proof. Clearly, if $f \in \mathrm{cl}(K)$, then $f(x) \leqslant f(y)$ when $g(x) \leqslant g(y)$ for all $g$ $\in K$. Now suppose $f \in C(X)$ is such that $f(x) \leqslant f(y)$ whenever $g(x) \leqslant g(y)$ for all $g \in K$. Let $\delta$ be the quasi-proximity defined by $K$. By the Characterization Theorem $\operatorname{cl}(K)=C(X, \delta)$, and so we must show that inf $f[A] \leqslant \sup f[B]$ whenever $A \delta B$. Accordingly, let $A \delta B$. By 5.8 there exist $x \in \operatorname{cl}(A)$ and $y \in \operatorname{cl}(B)$ such that $\{x\} \delta\{y\}$. By 4.1(ii) $g(x) \leqslant g(y)$ for all $g \in K$, and so $f(x) \leqslant f(y)$.

$$
\inf f[A]=\inf f[\operatorname{cl}(A)] \leqslant f(x) \leqslant f(y) \leqslant \sup f[\operatorname{cl}(B)]=\sup f[B] .
$$

5.10. Corollary. A compact ordered topological space has at most one compatible quasi-proximity.

Proof. Let $X$ be a compact ordered topological space and let $\delta$ be a compatible quasi-proximity on $X$. Let $f \in C(X, \leqslant)$ and let $x, y \in X$ be such that $g(x) \leqslant g(y)$ for all $g \in C(X, \delta)$. By 4.1(ii) $\{x\} \delta\{y\}$, i.e. $x \leqslant y$ and so $f(x) \leqslant f(y)$. 
Thus $f \in C(X, \delta)$ and so $\delta$ is the quasi-proximity defined by $C(X, \preccurlyeq)$.

Thus if a compact ordered topological space $X$ is to have a compatible quasi-proximity, the quasi-proximity $\delta$ defined by $C(X, \leqslant)$ must be compatible. We shall deduce the compatibility of $\delta$ from the following interposition theorem.

5.11. TheOREM. Let $(X, \leqslant)$ be a compact ordered topological space, and let $L(X, \lessgtr)$ (resp. $U(X, \preccurlyeq)$ ) be the set of bounded, increasing lower (resp. upper) semicontinuous functions on $X$. Then $C(X, \preccurlyeq)$ interposes $(U(X, \preccurlyeq), L(X, \preccurlyeq))$.

Proof. One easily sees that $U(X, \Im)=\overline{\operatorname{cn}}(A)$ and $L(X, \preccurlyeq)=\overline{\operatorname{cn}}(B)$, where $A$ and $B$ are, respectively, the set of closed increasing $\left(A \subset X\right.$ is increasing iff $1_{A}$ is increasing) subsets of $X$ and the open increasing subsets of $X$. Obviously, $C(X, \xi)=\overline{\operatorname{cn}}(A) \cap \overline{\operatorname{cn}}(B)$, and so we may apply 3.8. Accordingly, let $A \in A$, $B \in B$, and let $A \subset B$. Let $U$ be an open subset of $X$ and let $F$ be a closed subset of $X$ such that $A \subset U \subset F \subset B$. Set $A^{\prime}=\{x \in X: \exists y \in F \ni y \leqslant x\}$, and set $B^{\prime}=\{x \in X: x \leqslant y \Rightarrow y \in U\}$. Clearly, $A^{\prime}$ and $B^{\prime}$ are increasing, and $A \subset$ $B^{\prime} \subset U \subset F \subset A^{\prime} \subset B$. We must show that $A^{\prime}$ and $X \sim B^{\prime}$ are closed. Let $\left\{x_{i}\right\}_{i \in I}$ be a net in $A^{\prime}$ (resp., $X \sim B^{\prime}$ ) which converges to $x$. For each $i \in I$, let $y_{i} \in F$ (resp., $y_{i} \in X \sim U$ ) be such that $y_{i} \leqslant x_{i}$ (resp., $x_{i} \leqslant y_{i}$ ). Let $y$ be a cluster point of $\left\{y_{i}\right\}_{i \in I}$. Then $y \leqslant x$ (resp., $x \leqslant y$ ) and $y \in F$ (resp., $y \in X \sim U$ ) and so $x \in A^{\prime}$ (resp., $x \in X \sim B^{\prime}$ ).

5.12. Corollary. The quasi-proximity defined by $C(X, \preccurlyeq)$ is compatible.

Proof. Let $\tau$ be the topology of $X$, and let $\delta$ be the quasi-proximity defined by $C(X, \preccurlyeq) . \tau \supset \tau(\delta)$, and so we shall have that $\tau=\tau(\delta)$ once it is shown that $\tau(\delta)$ is Hausdorff, i.e., that $C(X, \leqslant)$ is separating. Now, it is enough to show that $x \leqslant y$ whenever $f(x) \leqslant f(y)$ for all $f \in C(X, \leqslant)$. Of course, this will also show that $\leqslant$ and $\leqslant_{\delta}$ are the same. Accordingly, let $x \notin y$. Set $A=\{z \in X$ : $x \leqslant z\}$, and set $B=\{z \in X: z \leqslant y\} .1_{A}$ is upper semicontinuous and increasing, $1_{X \sim B}$ is lower semicontinuous and increasing, and $1_{A} \leqslant 1_{X \sim B}$. By 5.11 there is an $f \in C\left(X, \leqslant\right.$ such that $1_{A} \leqslant f \leqslant 1_{X \sim B} . f(x)=1>0=f(y)$.

It will be convenient to have a description of $\delta$ which does not involve $C(X, \lessgtr)$. This is done via the Kuratowski closure operators $\mathrm{cl}_{i}$ and $\mathrm{cl}_{d}$ defined as follows.

5.13. Definition. Let $(X, \preccurlyeq)$ be an ordered topological space. For $A \subset X$, let $\mathrm{cl}_{i}(A)$ (resp., $\left.\mathrm{cl}_{d}(A)\right)$ be the intersection of all closed increasing (resp., decreasing) subsets of $X$ which contain $A$.

5.14. TheOREM. Let $(X, \$)$ be a compact ordered topological space. Then the compatible quasi-proximity $\delta$ on $X$ is given by

$$
A \delta B \Leftrightarrow \mathrm{cl}_{i}(A) \cap \mathrm{cl}_{d}(B) \neq \varnothing .
$$


Proof. $\Leftrightarrow$ Let $f \in C(X, \leqslant)$, and set $C=\{x \in X: f(x) \geqslant \inf f[A]\}$, $D=\{x \in X: f(x) \leqslant \sup f[B]\} . A \subset C$ and $C$ is a closed increasing subset of $X$ so that $\mathrm{cl}_{i}(A) \subset C$. Likewise, $\mathrm{cl}_{d}(B) \subset D$. Thus $C \cap D \neq \varnothing$, and so there is a $y \in X$ such that inf $f[A] \leqslant f(y) \leqslant \sup f[B]$. By 4.1(ii) $A \delta B$.

$\Leftrightarrow 1_{\mathrm{cl}}(A)$ is increasing and upper semicontinuous, $1_{X \sim \mathrm{cl}_{d}(B)}$ is increasing and lower semicontinuous, and $1_{\mathrm{cl}_{i}(A)} \leqslant 1_{X \sim \mathrm{cl}_{d}(B)}$, and so by 5.11 there is an $f \in C(X, \leqslant)$ such that $1_{\operatorname{cl}_{i}(A)} \leqslant f \leqslant 1_{X \sim \mathrm{cl}_{d}(X)} . f$ is 1 on $A$ and 0 on $B$ so that $A \bar{\delta} B$.

Now let $X$ be an ordered topological space, and let $(Y, \kappa)$ be an order compactification of $X$. Let $\eta$ be the compatible quasi-proximity on $Y$, and define the binary relation $\delta$ on $2^{X}$ by

$$
A \delta B \Longleftrightarrow \kappa[A] \eta \kappa[B] .
$$

We shall see that $\delta$ is a compatible quasi-proximity on $X$ and that $((Y, \eta), \kappa)$ is a compactification of $(X, \delta)$. This latter fact will be used to show that, up to equivalence, $(Y, \kappa)$ is determined by $\delta$. Note that an ordered topological space has an order compactification iff it has a compatible quasi-proximity, and by 5.7(iii) an ordered topological space $X$ has a compatible quasi-proximity iff the quasi-proximity defined by $C(X, \leqslant)$ is compatible. These considerations lead us to the following

5.15. Definition. An ordered topological space $(X, \tau, \preccurlyeq)$ is a completely regular ordered topological space if

- $\tau$ is the smallest topology on $X$ which renders continuous the members of $C(X, \leqslant)$,

- for $x, y \in X, x \leqslant y$ iff $f(x) \leqslant f(y)$ for all $f \in C(X, \leqslant)$.

Completely regular ordered topological spaces were introduced by Nachbin who proved that they are those spaces which have order compactifications [15, Appendix, §2].

5.16. Order COMpactification Theorem. Let $X$ be an ordered topological space.

(i) $X$ has an order compactification iff it is a completely regular ordered topological space.

(ii) If $(Y, \kappa)$ is an order compactification of $X$, then the binary relation $\delta$ on $2^{X}$ defined by

$$
A \delta B \Longleftrightarrow \mathrm{cl}_{i}(\kappa[A]) \cap \mathrm{cl}_{d}(\kappa[B]) \neq \varnothing
$$

is a compatible quasi-proximity on $X$ and is called the quasi-proximity associated with $(Y, \kappa)$.

(iii) If $\delta$ is a compatible quasi-proximity on $X$, then there is an order compactification of $X$ whose associated quasi-proximity is $\delta$. 
(iv) Two order compactifications of $X$ are equivalent iff they have the same associated quasi-proximity.

Proof. (i) The 'if' part of (i) follows from (iii) and the fact that the quasi-proximity defined by $C(X, \leqslant$ is compatible. The 'only if part of (i) follows from (ii) and 5.7(iii).

(ii) Let $\eta$ be the quasi-proximity on $Y$ defined by

$$
A \eta B \Longleftrightarrow \mathrm{cl}_{i}(A) \cap \mathrm{cl}_{d}(B) \neq \varnothing \text {. }
$$

By $5.14 \eta$ is compatible, and by $5.10 C(Y, \eta)=C(Y, \preccurlyeq)$. Thus for $A, B \subset X$, $A \delta B$ iff $\inf (f \circ \kappa)[A] \leqslant \sup (f \circ \kappa)[B]$ for all $f \in C(Y, \leqslant$, i.e. $\delta$ is the quasiproximity defined by the lattice cone $K=\{f \circ k: f \in C(Y, \leqslant)\}$. Since $k[X]$ is dense in $Y$, the mapping $f \mapsto f \circ \kappa$ of $C(Y, \leqslant$ to $C(X, \leqslant)$ is distance-preserving and so $K$ is closed. Thus $K=C(X, \delta)$. By (ii) of the Császár Compactification Theorem $((Y, \eta), \kappa)$ is a compactification of $(X, \delta)$. Since $\kappa$ is injective, it is, by 4.11 , a topological embedding with respect to $\tau(\delta)$ and $\tau(\eta)$. Since $\kappa$ is also a topological embedding with respect to the topology of $X$ and $\tau(\eta), \tau(\delta)$ is the topology of $X$. For $x, y \in X$,

$$
x \leqslant y \Longleftrightarrow \kappa(x) \leqslant \kappa(y) \Longleftrightarrow\{\kappa(x)\} \eta\{\kappa(y)\} \Longleftrightarrow\{x\} \delta\{y\} \Longleftrightarrow x \leqslant_{\delta} y .
$$

Thus $\delta$ is compatible.

(iii) Let $\delta$ be a compatible quasi-proximity on $X$. Let $((Y, \eta), \kappa)$ be a compactification of $(X, \delta) .\left(\left(Y, \tau(\eta), \leqslant_{\eta}\right), k\right)$ is an order compactification of $X$ (see the remarks following 5.5), and its associated quasi-proximity is $\delta$.

(iv) Let $\left(Y_{1}, \kappa_{1}\right)$ and $\left(Y_{2}, \kappa_{2}\right)$ be order compactifications of $X$ which have the same associated quasi-proximity, $\delta$. Let $\eta_{i}$ be the compatible quasi-proximity on $Y_{i}, i=1,2$. In the proof of (ii) we saw that $\left(\left(Y_{i}, \eta_{i}\right), \kappa_{i}\right)$ is a compactification of $(X, \delta), i=1,2$. By (iii) of the Császár Compactification Theorem there is a bijection $\varphi$ of $Y_{1}$ to $Y_{2}$ such that $\varphi \circ \kappa_{1}=\kappa_{2}$ and both $\varphi$ and $\varphi^{-1}$ are quasi-proximity mappings. Trivally, $\varphi$ is an order embedding, and by $4.8 \varphi$ is a homeomorphism.

5.17. Definition. An order compactification $(Y, k)$ of an ordered topological space $X$ is called a Nachbin compactification if its order is the smallest closed order on $Y$ with respect to which $k$ is an order embedding.

Nachbin compactifications are in a sense more "natural" than non-Nachbin compactifications. For example, if $X$ is a completely regular ordered topological space whose order is equality, then an order compactification $(Y, \kappa)$ of $X$ is a Nachbin compactification iff the order on $Y$ is equality.

We shall now describe the compatible quasi-proximities which are associated with Nachbin compactifications. 
5.18. Proposition. Let $X$ be an ordered topological space, and let $(Y, \kappa)$ be an order compactification of $X$. Set $K=\{f \in C(Y): f \circ \kappa \in C(X, \leqslant)\}$, and define the binary relation $\leqslant^{\prime}$ on $Y$ by

$$
x \leqslant{ }^{\prime} y \Longleftrightarrow f(x) \leqslant f(y) \text { for all } f \in K .
$$

Then $\leqslant$ ' is the smallest closed order on $Y$ with respect to which $k$ is an order embedding.

Proof. $\leqslant$ is clearly closed, transitive and reflexive. Since $K \supset C(Y, \leqslant)$, $\xi^{\prime}$ is also antisymmetric. For $x, y \in X$,

$$
\begin{aligned}
x \leqslant y & \Longleftrightarrow \kappa(x) \leqslant \kappa(y) \Longleftrightarrow(f \circ \kappa)(x) \leqslant(f \circ \kappa)(y) \text { for all } f \in K \\
& \Longleftrightarrow \kappa(x) \leqslant \kappa(y) .
\end{aligned}
$$

Thus $k$ is an order embedding with respect to $\leqslant^{\prime}$. Let $\leqslant^{\prime \prime}$ be a closed order on $X$ with respect to which $\kappa$ is an order embedding. $C\left(Y, \leqslant^{\prime \prime}\right) \subset K$ and so $x \leqslant$ $y \Rightarrow x \leqslant$ " $y$.

5.19. Corollary. If $\delta$ is the quasi-proximity associated with $(Y, \kappa)$, then $(Y, \kappa)$ is a Nachbin compactification iff $C(X, \delta)=\{f \circ \kappa: f \in C(Y)\} \cap C(X, \preccurlyeq)$.

Proof. If $(Y, \kappa)$ is a Nachbin compactification, then by the KakutaniStone Theorem $K=C(Y, \preccurlyeq)$, and so by (ii) of the Császár Compactification Theorem $C(X, \delta)=\{f \circ \kappa: f \in K\}=\{f \circ \kappa: f \in C(Y)\} \cap C(X, \preccurlyeq)$. Now suppose $C(X, \delta)=\{f \circ \kappa: f \in C(Y)\} \cap C(X, \leqslant)$. Let $x, y \in Y, x \leqslant y$. Let $f \in K$. $f \circ$ $\kappa \in C(X, \delta)$ and so $f \in C(Y, \leqslant$. Thus $f(x) \leqslant f(y)$. It follows that $x \leqslant y$. Hence $\leqslant$ ' and $\leqslant$ are the same.

It follows from 5.19 that once we have a description of $\{f \circ \kappa: f \in C(Y)\}$ solely in terms of $\delta$ we shall have a condition on $\delta$ necessary and sufficient for $(Y, \kappa)$ to be a Nachbin compactification. Such a description is provided by

5.20. Proposition. Let $X$ be an ordered topological space, $(Y, \kappa)$ an order compactification of $X$, and let $\delta$ be the associated quasi-proximity. Then $\{f \circ \kappa$ : $f \in C(Y)\}=\operatorname{cl}(C(X, \delta)-C(X, \delta))$.

Proof. First, we prove that $V=C(X, \delta)-C(X, \delta)$ is a vector sublattice of $B(X)$. Clearly, $V$ is a linear subspace of $B(X)$. Let $f, g \in V$, say $f=f_{1}-f_{2}, g=$ $g_{1}-g_{2} . \quad f \vee g=\left(\left(f_{1}+g_{2}\right) \vee\left(g_{1}+f_{2}\right)\right)-\left(f_{2}+g_{2}\right) \in V$ and $f \wedge g=$ $-((-f) \vee(-g)) \in V . \quad \mathrm{cl}(V)$ is a closed vector sublattice of $B(X)$ and so $L=$ $\{f \in C(Y): f \circ k \in \mathrm{cl}(V)\}$ is a closed vector sublattice of $C(Y)$. $L$ contains constant functions. By the Kakutani-Stone Theorem and the fact that $L=-L, L$ consists of $f \in C(Y)$ such that $f(x)=f(y)$ whenever $x, y \in Y$ and $g(x)=g(y)$ for all $g \in L$. $L \supset C(Y, \preccurlyeq$ and so if $g(x)=g(y)$ for all $g \in L$, then $x=y$. Thus $L=C(Y)$.

In view of 5.19 and 5.20 the following theorem is obvious. 
5.21. Nachbin Compactification Theorem I. Let $X$ be an ordered topological space, $(Y, \kappa)$ an order compactification of $X$, and let $\delta$ be the associated quasi-proximity. Then $(Y, \kappa)$ is a Nachbin compactification iff the only increasing members of $\operatorname{cl}(C(X, \delta)-C(X, \delta))$ are the members of $C(X, \delta)$.

We can reformulate 5.21 in such a way that no mention is made of $C(X, \delta)$. Among all proximities $\rho$ on $X$ (recall that a proximity is a symmetric quasi-proximity) with the property that $A \delta B$ (and $B \delta A$ ) whenever $A \rho B$, there is a largest, the symmetrization of $\delta$, which we denote by $\delta^{s}$. Namely, $\delta^{s}$ is the quasi-proximity defined by $C(X, \delta)-C(X, \delta)$. It follows from the Characterization Theorem that $\operatorname{cl}(C(X, \delta)-C(X, \delta))=C\left(X, \delta^{\mathcal{S}}\right)$. In the following definition we give a condition on $\delta$ which will insure that $(Y, \kappa)$ is a Nachbin compactification.

5.22. Definition. Let $(X, \delta)$ be a quasi-proximity space. We shall say that $\delta$ has the property (*) iff $\eta=\delta$ whenever $\eta$ is a quasi-proximity on $X$ such that $\eta \subset \delta, \leqslant_{\eta} \supset \leqslant_{\delta}$, and $\eta^{s}=\delta^{s}$.

5.23. Nachbin Compactification Theorem II. Let $X$ be an ordered topological space, $(Y, \kappa)$ an order compactification of $X$, and let $\delta$ be the associated quasi-proximity on $X$. Then $(Y, \kappa)$ is a Nachbin compactification iff $\delta$ has the property (*).

Proof. Let $\eta$ be the quasi-proximity on $X$ defined by $C(X, \leqslant) \cap\{f \circ \kappa$ : $f \in C(Y)\}$. Since $C(X, \delta) \subset C(X, \eta) \subset C\left(X, \delta^{5}\right), \eta^{s}=\delta^{s}$ and $\eta \subset \delta$. Since $C(X, \delta) \subset C(X, \eta) \subset C(X, \lessgtr), \eta$ is compatible. Finally, $C(X, \delta) \subset C(X, \eta) \subset$ $C(X, \leqslant) \cap\{f \circ k: f \in C(Y)\}$. The theorem now follows.

It is possible to define $\delta^{s}$ without reference to $C(X, \delta)$ (see [6, p. 20]), but this description is, to say the least, cumbersome. It would be quite desirable to obtain a simple condition on $\delta$ which would be necessary and sufficient for $(Y, \kappa)$ to be a Nachbin compactification of $X$.

5.24. Definition. Let $X$ be an ordered topological space, and let $\left(Y_{1}, \kappa_{1}\right)$ and $\left(Y_{2}, \kappa_{2}\right)$ be order compactifications of $X$. We shall say that $\left(Y_{1}, \kappa_{1}\right)$ is greater than $\left(Y_{2}, \kappa_{2}\right)$ if there is a $\varphi: Y_{1} \rightarrow Y_{2}$ such that

- $\varphi$ is continuous,

- $\varphi$ is order-preserving, i.e. $x \leqslant y \Rightarrow \varphi(x) \leqslant \varphi(y)$,

- $\kappa_{2}=\varphi \circ \kappa_{1}$.

5.25. TheOREM. Let $X$ be an ordered topological space.

(i) If $\left(Y_{i}, \kappa_{i}\right)$ is an order compactification of $X$ with associated quasiproximity $\delta_{i}, i=1,2$, then $\left(Y_{1}, \kappa_{1}\right)$ is greater than $\left(Y_{2}, \kappa_{2}\right)$ iff $\delta_{1} \subset \delta_{2}$.

(ii) If $K$ is a nonempty set of order compactifications of $X$, then there is an order compactification of $X$ which is greater than every member of $K$ and is smallest among all such compactifications. 
Proof. (i) Suppose $\delta_{1} \subset \delta_{2}$. Let $\eta_{i}$ be the compatible quasi-proximity on $Y_{i}, i=1$, 2. $A \delta_{1} B \Rightarrow A \delta_{2} B \Rightarrow \kappa_{2}[A] \eta_{2} \kappa_{2}[B]$ and so $\kappa_{2}$ is a quasi-proximity mapping of $\left(X, \delta_{1}\right)$ to $\left(Y_{2}, \eta_{2}\right)$ and so by (ii) of the Császár Compactification Theorem there is a quasi-proximity mapping $\varphi: Y_{1} \rightarrow Y_{2}$ such that $k_{2}=$ $\varphi \circ \kappa_{1} . \varphi$ is a continuous order-preserving mapping. Thus $\left(Y_{1}, \kappa_{1}\right)$ is greater than $\left(Y_{2}, \kappa_{2}\right)$. Now suppose we are given that $\left(Y_{1}, \kappa_{1}\right)$ is greater than $\left(Y_{2}, \kappa_{2}\right)$. Let $\varphi: Y_{1} \rightarrow Y_{2}$ be continuous, order-preserving and such that $\kappa_{2}=\varphi \circ \kappa_{1}$. Let $A \bar{\delta}_{2} B$. By the Characterization Theorem and by (ii) of the Császár Compactification Theorem there is an $f \in C\left(Y_{2}, \preccurlyeq\right)$ such that $f \circ \kappa_{2}$ is 1 on $A$ and 0 on $B$. $f \circ \varphi \in C\left(Y_{1}, \preccurlyeq\right.$ and $f \circ \varphi$ is 1 on $\kappa_{1}[A]$ and 0 on $\kappa_{1}[B]$, i.e. $A \bar{\delta}_{1} B$. Thus $\delta_{1} \subset \delta_{2}$.

(ii) Let $\Delta$ be the set of all quasi-proximities on $X$ which are associated with members of $K$. Let $K_{0}$ be the intersection of all closed lattice cones on $X$ which contain $\bigcup_{\delta \in \Delta} C(X, \delta)$, and let $\delta_{0}$ be the quasi-proximity defined by $K_{0}$. $\delta_{0}$ is compatible and any order compactification associated with $\delta_{0}$ will have the desired properties.

6. An approximation theorem. This section is devoted to proving the Approximation Theorem ( 6.2 below) and to deriving as a corollary an extension of a well-known result of Nachbin [15, Appendix, $\S 5$, Theorem 6]. A different proof for part of the Approximation Theorem has been given in [3].

6.1. Definition. Let $X$ be a set, $S$ a nonvoid subset of $B(X)$, and let $f \in$ $B(X)$. The distance of $f$ to $S$ is defined to be the number $\operatorname{dist}(f, S)=\inf \{\|f-g\|$ : $g \in S\}$.

6.2. Approximation Theorem. Let $X$ be a set, $X_{0}$ a subset of $X, K a$ closed lattice cone on $X, \delta$ the quasi-proximity defined by $K$, and let $K_{0}=\{f \in$ $\left.K: f \mid X_{0}=0\right\}$. Then for $f \in B(X)$, dist $\left(f, K_{0}\right)$ is the largest of the numbers $\sup \left\{1 \frac{1}{2}(\inf f[A]-\sup f[B]): A \delta B\right\}$,

$\sup \left\{\inf f[A]: A \delta X_{0}\right\}$, $\sup \left\{-\sup f[B]: X_{0} \delta B\right\},\left(^{7}\right)$

and there is a $k_{0} \in K_{0}$ such that $\left\|f-k_{0}\right\|=\operatorname{dist}\left(f, K_{0}\right)$.

Proof. Set $d=\operatorname{dist}\left(f, K_{0}\right)$, and let $m$ be the largest of the above numbers. We first show that $d \geqslant m$ and then complete the proof by showing the existence of a $k_{0} \in K_{0}$ such that $\left\|f-k_{0}\right\| \leqslant m$. Let $k \in K_{0}$, and set $d^{\prime}=$ $\|k-f\|$. We must show that $d^{\prime} \geqslant m$. If $A \delta B$, then by $4.1(\mathrm{i})$, inf $k[A] \leqslant$ $\sup k[B]$ so that

$$
\inf f[A]-d^{\prime} \leqslant \inf k[A] \leqslant \sup k[B] \leqslant \sup f[B]+d^{\prime},
$$

(7) In the case that $x_{0}=\varnothing$, the two suprema involving $x_{0}$ are taken to be 0 . 
i.e. $2 d^{\prime} \geqslant \inf f[A]-\sup f[B]$. Thus

$$
d^{\prime} \geqslant \sup \{\not / 2(\inf f[A]-\sup f[B]): A \delta B\} .
$$

If $A \delta X_{0}$, then again by 4.1(i), $\inf k[A] \leqslant \sup k\left[X_{0}\right]=0$ and so inf $f[A]-d^{\prime}$ $\leqslant \inf k[A] \leqslant 0$, i.e. $d^{\prime} \geqslant \inf f[A]$. Thus $d^{\prime} \geqslant \sup \left\{\inf f[A]: A \delta X_{0}\right\}$. Likewise one sees that $d^{\prime} \geqslant \sup \left\{-f[B]: X_{0} \delta B\right\}$. Thus $d^{\prime} \geqslant m$. It follows that $d \geqslant m$. Now we show the existence of $k_{0}$. Define $f_{*}, f^{*} \in B(X)$ by

$$
f_{*}(x)=\left\{\begin{array}{ll}
f(x)-m, & x \in X \sim X_{0}, \\
0, & x \in X_{0},
\end{array} f^{*}(x)= \begin{cases}f(x)+m, & x \in X \sim X_{0}, \\
0, & x \in X_{0} .\end{cases}\right.
$$

Suppose for the moment we have a $k \in K$ such that $f_{*} \leqslant k \leqslant f^{*}$. Certainly, $k$ $\in K_{0}$. We claim that $\|f-k\| \leqslant m$. Clearly, $|f-k| \leqslant m$ on $X \sim X_{0}$. Let $x \in$ $X_{0} .\{x\} \delta X_{0}$ implies that $f(x) \leqslant m$, and $X_{0} \delta\{x\}$ implies that $-f(x) \leqslant m$. Thus $|f-k| \leqslant m$ on $X_{0}$. Consequently, we need only find such a $k$, and to this end it suffices, by the Interposition Theorem, to show that $f_{*} \prec_{K} f^{*}$. Let $r, s \in$ $\mathrm{R}, r<s$. Suppose it is false that $\left\{x: f_{*}(x) \geqslant s\right\} \prec_{K}\left\{x: f^{*}(x) \geqslant r\right\}$, i.e. $\{x$ : $\left.f_{*}(x) \geqslant s\right\} \delta\left\{x: f_{*}(x)<r\right\}$. Since $|f(x)| \leqslant m$ for $x \in X_{0}$, we have

$$
\begin{aligned}
& \left\{x: f_{*}(x) \geqslant s\right\}= \begin{cases}\{x: f(x) \geqslant s+m\} \cup X_{0}, & s \leqslant 0, \\
\{x: f(x) \geqslant s+m\}, & s>0,\end{cases} \\
& \left\{x: f^{*}(x)<r\right\}= \begin{cases}\{x: f(x)<r-m\}, & r \leqslant 0, \\
\{x: f(x)<r-m\} \cup X_{0}, & r>0 .\end{cases}
\end{aligned}
$$

Set $A=\{x: f(x) \geqslant s+m\}, B=\{x: f(x)<r-m\}$. We consider, in order, the cases $r<s \leqslant 0, r \leqslant 0<s, 0<r<s$.

$r<s \leqslant 0$. $\left(A \cup X_{0}\right) \delta B$ and so $A \delta B$ or $X_{0} \delta B$. In case $A \delta B$, we have

$$
m \geqslant 1 / 2(\inf f[A]-\sup f[B]) \geqslant 1 / 2(s+m)=m+1 / 2(s-r)>m,
$$

and in case $X_{0} \delta B$ we have

$$
m \geqslant-\sup f[B] \geqslant-r+m>m .
$$

This disposes of the case that $r<s \leqslant 0$.

$r \leqslant 0<s$. In this case we have $A \delta B$ which, as we have already seen, leads to a contradiction. So much for the case $r \leqslant 0<s$.

$0<r<$ s. $A \delta\left(B \cup X_{0}\right)$ and so $A \delta B$ or $A \delta X_{0}$. We have already seen that $A \bar{\delta} B$. Thus $A \delta X_{0} . \quad m \geqslant \inf f[A] \geqslant s+m$, our final contradiction.

6.3. Corollary. If $X$ is a compact topological space, $K \subset C(X)$, and if 
$f \in C(X)$, then $\operatorname{dist}\left(f, K_{0}\right)$ is the largest of the numbers

$\sup \{1 / 2(f(x)-f(y)):\{x\} \delta\{y\}\}$,

$\sup \left\{f(x):\{x\} \delta X_{0}\right\}$,

$\sup \left\{-f(x): X_{0} \delta\{x\}\right\}$.

Proof. By the theorem the largest of the above numbers does not exceed $\operatorname{dist}\left(f, K_{0}\right)$. Let $A \delta B$. By the Translation Lemma, 5.8, there exist $x \in \operatorname{cl}(A)$ and $y \in \operatorname{cl}(B)$ such that $\{x\} \delta\{y\} . f(x)-f(y) \geqslant \inf f[A]-\sup f[B]$. Thus

$$
\sup \{1 / 2(f(x)-f(y)):\{x\} \delta\{y\}\}=\sup \{1 / 2(\inf f[A]-\sup f[B]): A \delta B\} .
$$

Likewise we see that

$$
\sup \left\{f(x):\{x\} \delta X_{0}\right\}=\sup \left\{\inf f[A]: A \delta X_{0}\right\}
$$

and

$$
\sup \left\{-f(x): X_{0} \delta\{x\}\right\}=\sup \left\{-\inf f[B]: X_{0} \delta B\right\} .
$$

6.4. Corollary (Nachbin [15, Appendix, §5, Theorem 6]). Let $X$ be a compact topological space, $K$ a closed lattice cone of continuous functions on $X$, and let $f \in C(X)$. Then

$$
\operatorname{dist}(f, K)=\sup \{1 / 2(f(x)-f(y)): x, y \in X, k(x) \leqslant k(y) \text { all } k \in K\},
$$

and there is a $k \in K$ such that $\operatorname{dist}(f, K)=\|f-k\|$.

6.5. Corollary. If $X$ is a set, and if $A$ is a closed subalgebra of $B(X)$, with or without identity, then for $f \in B(X)$, there is a $g \in A$ such that $\operatorname{dist}(f, A)$ $=\|f-g\|$.

Proof. The hypotheses of 6.2 are satisfied by $K=A+\mathbf{R} \cdot 1_{X}$ and $X_{0}=$ $\{x: g(x)=0$ for all $g \in A\}$. In this case $K_{0}=A$.

In connection with 6.5 see [16], [12], [13], and [1, Chapter 3]. It is shown in $[1,3.22-3.25]$ that the condition " $A$ is a closed subalgebra of $B(X)$ " of 6.5 cannot be replaced by the condition " $A$ is a closed vector sublattice of $B(X)$ "'.

\section{REFERENCES}

1. J. Blatter, Grothendieck spaces in approximation theory, Mem. Amer. Math. Soc. No. 120 (1972).

2. J. Blatter and G. L. Seever, Interposition of semi-continuous functions by continuous functions, Analyse Fonctionelle et Applications (Comptes Rendus du Colloque d'Analyse, Rio de Janeiro, 1972), Hermann, Paris, 1975, pp. 27-51.

3. Inclusions, interposition and approximation, Approximation Theory, G. G. Lorentz, ed., Academic Press, New York, 1973, pp. 257-261.

4. Quasi-proximities and order compactifications, Notices Amer. Math. Soc. 20 (1973), A-594. Abstract \#73T-G122. 
5. A. Calder, Proximity algebras, Carleton Math. Ser., no. 44, Carleton University, Ottawa, Canada.

6. A. Császár, Foundations of general topology, Macmillan, New York, 1963. MR 28 \#575.

7. C. H. Dowker, On countably paracompact spaces, Canad. J. Math. 3 (1951), $219-$ 224. MR 13, 264.

8. V. A. Efremovič, Infinitesimal spaces, Dokl. Akad. Nauk SSSR 76 (1951), 341 343. (Russian) MR 12, 744.

9. The geometry of proximity. I, Mat. Sb. 31 (73) (1952), 189-200. (Russian) MR 14, 1106.

10. M. Katětov, On real-valued functions in topological spaces, Fund. Math. 38 (1951), 85-91. MR 14, 304.

11. Correction to "On real-valued functions in topological spaces", Fund. Math. 40 (1953), 203-205. MR 15, 640.

12. B. R. Kripke and R. B. Holmes, Approximation of bounded functions by continuous functions, Bull. Amer. Math. Soc. 71 (1965), 896-897. MR 32 \#185.

13. - Interposition and approximation, Pacific J. Math. 24 (1968), 103-110.

MR 36 \#4230.

14. E. A. Michael, Continuous selections. I, Ann. of Math. (2) 63 (1956), 361-382. MR 17, 990.

15. L. Nachbin, Topology and order, Van Nostrand Math. Studies, no. 4, Van Nostrand, Princeton, N. J., 1965. MR 36 \#2125.

16. A. Pefczyński, A generalisation of Stone's theorem on approximation, Bull. Acad.

Polon. Sci. Cl. III 5 (1957), 105-107. MR 19, 135.

17. W. J. Pervin, Quasi-proximities for topological spaces, Math. Ann. 150 (1963), 325-326. MR 27 \#1917.

18. F. Riesz, Stetigskeitsbegriff und abstrakte Mengenlehre, Atti del IV Congresso Intern. dei Matem., Roma, 1908, v. 11, Rome, 1909, pp. 18-24.

19. Ju. M. Smirnov, On proximity spaces in the sense of $V$. A. Efremovič, Dokl. Akad. Nauk SSSR 84 (1952), 895-898; English transl., Amer. Math. Soc. Transl. (2) 38 (1964), 1-4. MR 14, 1107.

20. On proximity spaces, Mat. Sb. 31 (73) (1952), 543-574; English transl., Amer. Math. Soc. Transl. (2) 38 (1964), 5-35. MR 14, 1107.

21. E. F. Steiner, The relation between quasi-proximities and topological spaces,

Math. Ann. 155 (1964), 194-195. MR 29 \#581.

22. M. H. Stone, Boundedness properties in function-lattices, Canad. J. Math. 1 (1949), 176-186. MR 10, 546.

23. H. Tong, Some characterizations of normal and perfectly normal spaces, Bull. Amer. Math. Soc. 54 (1948), 65.

24. Some characterizations of normal and perfectly normal spaces, Duke Math. J. 19 (1952), 289-292. MR 14, 304.

INSTITUT FÜR ANGEWANDTE MATHEMATIK UNIVERSITÄT BONN, 5300 BONN, FEDERAL REPUBLIC OF GERMANY

DEPARTMENT OF MATHEMATICS, UNIVERSITY OF TEXAS, AUSTIN, TEXAS 78712 (Current address of G. L. Seever)

INSTITUTO DE MATEMÁTICA, UNIVERSIDADE FEDERAL DO RIO DE JANEIRO, CAIXA POSTAL 1835, ZC-00, 20000 RIO DE JANEIRO, GB, BRAZIL

Current address (Jörg Blatter): Instituto de Matemática Pura e Aplicada, Rua Lutz de Camões 68, Rio de Janeiro, Brazil 